\title{
Carbohydrate-dependent $B$ cell activation by fucose-binding bacterial lectins
}

\author{
Isabel Wilhelm ${ }^{1,2,3^{*}}$, Ella Levit-Zerdoun ${ }^{4,5,6^{*}}$, Johanna Jakob ${ }^{7}$, Sarah Villringer ${ }^{1,3}$, Marco \\ Frensch $^{1,3,5}$, Rudolf Übelhart ${ }^{7}$, Alessia Landi ${ }^{1,3}$, Peter Müller ${ }^{1,3}$, Anne Imberty ${ }^{8}$, Roland \\ Thuenauer $^{1,3}$, Julie Claudinon ${ }^{1,3}$, Hassan Jumaa ${ }^{7}$, Michael Reth ${ }^{1,3,4}$, Hermann Eibel ${ }^{9,10}$, Elias \\ Hobeika $^{7 \dagger \dagger}$ and Winfried Römer ${ }^{1,2,3 \dagger \ddagger}$
}

${ }^{1}$ Faculty of Biology, University of Freiburg, 79104 Freiburg, Germany; ${ }^{2}$ Spemann Graduate School of Biology and Medicine (SGBM), University of Freiburg, 79104 Freiburg, Germany; ${ }^{3}$ BIOSS-Centre for Biological Signalling Studies, University of Freiburg, 79104 Freiburg, Germany; ${ }^{4}$ Max Planck Institute of Immunology and Epigenetics Freiburg, 79108 Freiburg, Germany; ${ }^{5}$ International Max Planck Research School (IMPRS), Max Planck Institute of Immunobiology and Epigenetics Freiburg, 79108 Freiburg, Germany; ${ }^{6}$ German Cancer Consortium (DKTK) partner site Freiburg, German Cancer Center (DKFZ), Heidelberg, Institute of Molecular Medicine and Cell Research, 79104 Freiburg, Germany; ${ }^{7}$ Institute for Immunology, University Medical Centre Ulm, 89081 Ulm, Germany; ${ }^{8}$ Univ. Grenoble Alpes, CNRS, CERMAV, 38000 Grenoble, France; ${ }^{9}$ CCI-Center for Chronic Immunodeficiency (CCI), University Medical Centre, 79106 Freiburg, Germany; ${ }^{10}$ Faculty of Medicine, University of Freiburg, 79106 Freiburg, Germany.

*These authors contributed equally to this work.

${ }^{\dagger}$ These senior authors contributed equally to this work.

†Corresponding author. Email: elias.hobeika@uni-ulm.de (E.H.); winfried.roemer@bioss.unifreiburg.de (W.R.)

\begin{abstract}
Bacterial lectins are typically multivalent and bind noncovalently to specific carbohydrates on host tissues to facilitate bacterial adhesion. Here, we analyzed the effects of two fucose-binding lectins, BambL from Burkholderia ambifaria and LecB from Pseudomonas aeruginosa, on specific signaling pathways in B cells. We found that these bacterial lectins induced B cell activation, which, in vitro, was dependent on the cell surface expression of the B cell antigen receptor (BCR) and its coreceptor CD19, as well as on spleen tyrosine kinase (Syk) activity. The resulting release of intracellular $\mathrm{Ca}^{2+}$ was followed by an increase in the cell surface abundance of the activation marker CD8, increased cytokine secretion, and subsequent cell death, replicating all of the events that are observed in vitro upon canonical and antigen-mediated B cell activation. Moreover, injection of BambL in mice resulted in a substantial, BCR-independent loss of B cells in the bone marrow with simultaneous, transient enlargement of the spleen (splenomegaly), as well as an increase in the numbers of splenic B cells and myeloid cells. Together, these data suggest that bacterial lectins can initiate polyclonal activation of B cells through their sole capacity to bind to fucose.
\end{abstract}




\section{Introduction}

Pseudomonas and Burkholderia species are proteobacteria, which release various lectins and form biofilms $(1,2)$. These bacteria cause opportunistic infections and exhibit multidrug antibiotic resistance (2-4). B. ambifaria belongs to the Burkholderia cepacia complex, a group of closely related bacterial strains, which cause socalled cepacia syndrome (4). The fatal combination of necrotizing pneumonia, respiratory failure, and bacteremia has been observed in the lung infections of patients suffering from cystic fibrosis (CF), as well as in nosocomial infections of other immunocompromised hosts (5-7). Furthermore, $B$. ambifaria species were identified in a polyclonal bacteremia outbreak caused by contaminated ultrasound gel in Brazil in 2015, but the epidemiology of $B$. ambifaria remains poorly characterized to date (7).

In addition to many other virulence factors, these bacteria produce soluble, carbohydrate-binding proteins, so-called lectins. $P$. aeruginosa expresses two tetravalent lectins, LecA and LecB (also called PA-IL and PA-IIL), with carbohydrate-binding specificities for galactose and fucose, respectively (8). The B. ambifaria lectin BambL (gene annotation across databases: bamb_5415; uniprotID: Q0B4G1 BURCM; NCBI-ProteinID: ABI90962; KEGGG-ID: bam:Bamb_5415) contains six carbohydrate-binding sites (9). Both LecB and BambL display exceptionally high affinities for fucose, with the respective $K_{d}$ values in the micromolar range, which is rather unusual for a lectin-monosaccharide interaction (9). Studies point to an underestimated, highly complex role of bacterial lectins in affecting host cellular processes, going far beyond an adhesive function (10-14). In particular, there is very limited knowledge about the influence of soluble bacterial lectins on cells of the immune system $(15,16)$.

However, many receptors of immune cells, including the $\mathrm{B}$ cell antigen receptor (BCR), are highly glycosylated and thus present potential targets for lectins (17-19). The BCR, which is expressed by B lymphocytes, plays a pivotal role in the humoral immune response. In mammals, B lymphocyte development follows a strict, multistep maturation program starting in the bone marrow (BM) from hematopoietic stem cells through progenitor (pro-) to precursor (pre-) cell stages. The pre-B cells are defined by their expression of the pre-BCR and further develop into immature B cells, which mainly express a BCR of the immunoglobulin $M(\operatorname{IgM})$ isotype $(20,21)$. Subsequently, these immature B cells migrate to the periphery and secondary lymphoid organs such as the spleen and the lymph nodes, where they undergo further differentiation, eventually coexpressing IgM- and IgD-BCR isotypes of the same antigen specificity. IgD expression increases with progressing differentiation, with mature $\mathrm{B}$ cells expressing more $\operatorname{IgD}$ than $\operatorname{IgM}(20,22)$.

The BCR comprises two heavy chains (HCs), two light chains (LCs), and the $\operatorname{Ig} \alpha / \operatorname{Ig} \beta$ (CD79a/CD79b) heterodimer, which functions as the signaling component of the BCR complex (2325). Mature B cells also carry a co-receptor complex comprising the molecules CD19, CD81, and CD21 (26). After B cell activation, CD19 is found in close proximity to the IgM-BCR and signals through the phosphoinositide-3 kinase (PI3K) pathway (27). B cell survival and differentiation are also promoted by the B cellactivating factor of the TNF-family (BAFF), which is also known as B-Lys $(28,29)$. The BCR can be activated by monovalent and polyvalent antigens $(30,31)$. IgD-BCRs are specialized in the binding of multivalent antigens, whereas IgM-BCRs can bind to both types of antigen $(30,32,33)$.

The constant region of both IgM- and IgD-BCRs is $\mathrm{N}$-glycosylated with glycans of the complex type, whereas the pre-BCR is highly mannosylated (18). Fucosylated epitopes can be part of N-linked or Olinked sugar chains. However, due to the challenges in resolving the glycosylation patterns of the BCR with spectrometric techniques, the exact amounts and sites, particularly of O-linked glycans, have not been elucidated to date (34-36). Furthermore, the number and position of N-linked glycosylation sites may vary between murine and human BCRs (34, 36). However, there are indications that glycosylation events can affect B cell development in mice, wherein a specific glycosylation site $\left(\mathrm{Asn}^{46}, \mathrm{~N} 46\right)$ on IgM is required for efficient preBCR function and further B cell maturation (17). Furthermore, the glycosylation pattern of the BCR changes during cancerogenesis: follicular lymphoma (FL) cells acquire carbohydrates of the 
high mannose type in the variable domain of the $\mathrm{BCR}$, generating binding sites for mannose-binding lectins $(19,37)$. Moreover, the Ig $\alpha$ and whole BCR molecules carry altered glycans in chronic lymphocytic leukemia (CLL), influencing BCR signaling (38).

In this study, we found that the fucose-specific bacterial lectins BambL and LecB bound to murine $\mathrm{B}$ cells and induced an intracellular signaling cascade that changed the flux of $\mathrm{Ca}^{2+}$. In vitro, activation of this cascade by BambL required expression of the BCR, as well as of its co-receptor CD19. Stimulation by BambL induced canonical BCR-mediated signaling events that resulted in a polyclonal B cell death, which was partially reversed by BAFF-induced signaling. In vivo, intraperitoneal (i.p.) injection of BambL resulted in lymphopenia in the bone marrow combined with a transient splenomegaly, as well as accumulation of $\mathrm{B}$ cells and myeloid cells in the spleen. These events were independent of BCR signaling because they were recapitulated in BCR-deficient mice. We propose that these bacterial lectins act as superstimulatory antigens, activating B cells in a BCRdependent manner in vitro by binding to fucosylated epitopes and eliciting a strong, but transient, BCR-independent immune response in vivo.

\section{Results}

\section{BambL induces $\mathrm{Ca}^{2+}$ release that is dependent on BCR expression and Syk activity}

Upon antigen binding, the immunoreceptor tyrosine-based activation motifs (ITAMs) of the Ig $\alpha / \beta$ subunits are phosphorylated, activating an intracellular signaling cascade $(23,39)$. As a part of the second messenger cascade, the intracellular $\mathrm{Ca}^{2+}$ concentration changes by store-operated calcium entry (SOCE) through the $\mathrm{Ca}^{2+}$ releaseactivated $\mathrm{Ca}^{2+}$ (CRAC) channels $(40,41)$. To assess whether the two fucose-binding lectins, LecB and BambL, activated primary murine B cells, we performed intracellular $\mathrm{Ca}^{2+}$ flux measurements in vitro (Fig. 1). The hexavalent BambL $(10 \mu \mathrm{g} / \mathrm{ml})$ induced a $\mathrm{Ca}^{2+}$ release similar to that induced by BCR stimulation with anti-kappa antibodies $(10 \mu \mathrm{g} / \mathrm{ml})$ in mature follicular (MFo) B cells (Fig. 1A). Saturating the BambL carbohydrate binding sites with soluble L-Fucose $(25 \mathrm{mg} / \mathrm{ml})$ prevented BambL binding to B cells (fig. S1A) and $\mathrm{Ca}^{2+}$ flux
(Fig. 1A), indicating that this activation process was fucose-specific. The mitogenic potential of LecB on murine splenocytes was described previously (15). Here, we demonstrated that binding of the tetravalent LecB $(10 \mu \mathrm{g} / \mathrm{ml})$ to B cells also induced $\mathrm{a} \mathrm{Ca}^{2+}$ signal, but to a somewhat lesser extent than that induced by the hexavalent BambL (Fig. 1A).

To assess whether the strength of lectin binding correlated with the extent of $\mathrm{Ca}^{2+}$ flux, we analyzed the binding of both lectins by flow cytometry and found that both lectins bound to B cells. LecB is tetravalent with a molecular weight (MW) of $46.924 \mathrm{kDa}$, whereas BambL is hexavalent with a MW of $28.143 \mathrm{kDa}$. Due to its hexavalency, the avidity of BambL is increased, which resulted in higher binding capacity at similar concentrations in comparison to LecB (Fig. S1A). The binding capacity may be further influenced by the differences in BambL lectin structure in comparison to $\mathrm{LecB}$, as well as the affinity of BambL for different host cell receptors. Furthermore, the lower MW of BambL supposedly leads to the binding of more molecules per cell (Fig. S1A). With BambL and LecB showing similar effects on B cells, we focused on the characterization of BambL binding. Additional binding assays demonstrated that BambL bound to all CD19-positive splenic B cells, as well as T cells (Fig. S1B). Analyzing $\mathrm{Ca}^{2+}$ mobilization in response to BambL binding in terms of dosedependency, we found a clear correlation in the range between 0.1 and $10 \mu \mathrm{g} / \mathrm{ml}$ of the lectin (Fig. 1B). To dissect the role of individual components of the BCR-associated signaling cascade in the response to BambL, we analyzed $\mathrm{Ca}^{2+}$ mobilization using $\mathrm{B}$ cells from mice lacking spleen tyrosine kinase (Syk), the Src-family kinase Lyn, or the BCR HC. Syk and Lyn are the most proximal kinases to the $\mathrm{BCR}$ and are involved in canonical B cell activation $(25,43)$. Similar to the $\mathrm{Ca}^{2+}$ release upon BCR stimulation by anti-IgM $\mathrm{F}\left(\mathrm{ab}^{\prime}\right)_{2}$, the BambL-mediated $\mathrm{Ca}^{2+}$ release was independent of Lyn but required the presence of the kinase Syk (Fig. 1C). Note that BambL efficiently bound to Syk-deficient B cells (Fig. S1D). Stimulation with anti-IgM served as a control for $\mathrm{Ca}^{2+}$ release in these experiments; however, due to normalization of the $y$-axis to the BambL-mediated $\mathrm{Ca}^{2+}$ release experiments, we cannot directly 
compare our data to those from previous studies $(42,43)$.

In a similar approach, we monitored $\mathrm{Ca}^{2+}$ release in $B$ cells lacking surface $B C R$ expression using mice carrying a B1-8 HC allele flanked by loxP sites (B1$\left.8^{\mathrm{fl}}\right)$ on an inducible mb1-CreERT ${ }^{\mathrm{T} 2}$ "deleter" background $(44,45)$. These mice express a B cellspecific Cre recombinase from the Ig $\alpha$-encoding $m b-1$ locus, which upon tamoxifen treatment induces the deletion of the $\mathrm{B} 1-8^{\mathrm{fl}}$ allele, resulting in the generation of HC-deficient $\mathrm{B}$ cells. The HCdeficient B cells were obtained at day 7 after tamoxifen treatment when an abundant number of these cells was still present in mb1-CreER ${ }^{\mathrm{T} 2} ; \mathrm{B} 1-8^{\mathrm{fl}}$ mice before they were lost at later time points (4446). These cells (denoted as $\mathrm{HC}^{-/-}$cells), identified by gating on IgM- and IgD-negative populations (Fig. S1C), displayed no detectable $\mathrm{Ca}^{2+}$ flux when stimulated with BambL despite effectively binding BambL (Fig. 1D and fig. S1D). The efficiency of tamoxifen-induced Cre-mediated recombination is not $100 \%$ in the mb1-CreER ${ }^{\mathrm{T} 2}$; B1- $8^{\mathrm{fl}}$ mice. Only approximately $15-20 \%$ of B cells lost HC 7 days after tamoxifen treatment. Therefore, IgM- and IgD-positive B cells were still detected in these animals and served as an internal HC-sufficient control $\left(\mathrm{HC}^{+/+}\right.$cells; Fig. 1D and S1C). These data indicate that BambL-dependent activation of $\mathrm{B}$ cells requires the presence of the BCR and Syk to mobilize intracellular $\mathrm{Ca}^{2+}$ release and is abrogated in the presence of L-Fucose.

\section{BambL-induced $B$ cell activation is restricted to IgD-expressing B cells}

We next examined whether activation of $B$ cells by BambL differed between precursor and mature $\mathrm{B}$ cells. To this end, we compared BambL-triggered $\mathrm{Ca}^{2+}$ release in bone marrow-derived and splenic $\mathrm{B}$ cells. We stained bone marrow-derived and splenic B cells with antibodies to detect the expression of typical cell surface markers to distinguish among the different B cell maturation stages (fig. S2). In contrast to incubation of the cells with anti$\operatorname{IgM~F}\left(\mathrm{ab}^{\prime}\right)_{2}$, incubation with BambL did not result in $\mathrm{Ca}^{2+}$ flux in pre-B cells or immature $\mathrm{B}$ cells (Fig. 2A), although BambL bound to both subsets of cells (Fig. S2B). Similarly, transitional 1 (T1) B cells from the spleen did not release $\mathrm{Ca}^{2+}$, whereas splenic T2/T3, marginal zone (MZ), and MFo B cells displayed changes in intracellular $\mathrm{Ca}^{2+}$ concentrations upon treatment with BambL (Fig. 2A). Immature and $\mathrm{T} 1 \mathrm{~B}$ cells express little to no IgD (22) (Fig. S2D), therefore we suspected that the activation of mature $\mathrm{B}$ cells by BambL was IgDdependent. To test this hypothesis, we analyzed BambL-induced $\mathrm{Ca}^{2+}$ flux using bone marrowderived B cells from $\operatorname{IgM}^{-1-}$ mice (47). In these mice, the DNA sequences encoding the constant region of the $\mu \mathrm{HC}$ have been removed, enforcing the expression of IgD starting from early maturation stages. We observed that BambL increased intracellular $\mathrm{Ca}^{2+}$ concentrations in these $\mathrm{B}$ cells (Fig. 2B). These data demonstrate that the IgMBCR was not required for BambL-induced $\mathrm{Ca}^{2+}$ release and suggest that the $\operatorname{IgD}$, but not the $\operatorname{IgM}$, isotype of the BCR is required for BambL-induced $\mathrm{Ca}^{2+}$ release in mature $\mathrm{B}$ cells.

B cell blasts treated with the Toll-like receptor 4 (TLR4) agonist lipopolysaccharide (LPS) downregulate cell-surface IgD and differentiate into $\mathrm{IgM}^{\text {high }}$ plasmablasts (48). We therefore compared $\mathrm{Ca}^{2+}$ release in LPS-treated $\mathrm{B}$ cells isolated from $\mathrm{WT}$ and $\mathrm{IgM}^{-/}$mice, which had been crossed with Blimp-1 ${ }^{\mathrm{GFP}}$ mice. In the latter, a green fluorescent protein (GFP) reporter is expressed from the Prdm1 gene locus, which encodes the transcription factor Blimp-1, a master regulator of plasma cell development expressed at early stages of plasma cell differentiation $(49,50)$. Plasmablasts were identified based on the expression of GFP and the plasma cell marker Syndecan-1 (CD138). WT plasmablasts displayed only a moderate $\mathrm{Ca}^{2+}$ release upon exposure to BambL, whereas $\operatorname{IgM}^{-/}$ plasmablasts, which expressed only IgD, exhibited a greater intracellular $\mathrm{Ca}^{2+}$ response (Fig. 2C). These results further support our hypothesis that only IgD-bearing B cells are activated by BambL.

\section{BambL-induced murine $B$ cell activation also requires CD19}

To corroborate our findings that BambL mobilized $\mathrm{Ca}^{2+}$ only in $\mathrm{IgD}^{+} \mathrm{B}$ cells, we compared changes in $\mathrm{Ca}^{2+}$ concentration in IgM- and in IgD-deficient $\mathrm{B}$ cells after incubation with different concentrations of BambL. As expected, we observed that the $\mathrm{IgD}^{-/-} \mathrm{B}$ cells expressing only IgM (Fig. S3) were able to bind to BambL but failed to mobilize $\mathrm{Ca}^{2+}$, which was in contrast to the responses of WT B cells and B cells expressing IgD alone (Fig. 3A and $\mathrm{B})$. Because the IgD-BCRs of resting mature $\mathrm{B}$ 
lymphocytes are reportedly organized in "protein islands" with the co-receptor CD19 in close proximity $(51,52)$, we assumed that the IgDdependent activation of $\mathrm{B}$ cells by BambL might also involve CD19. Although CD19-deficient B cells boudn to BambL to a similar extent as did WT $\mathrm{B}$ cells, they released much less $\mathrm{Ca}^{2+}$ than did WT B cells upon exposure to BambL (Fig. 3C and D). $\mathrm{B}$ cells from mice lacking both $\mathrm{IgD}$ and CD19 were still able to bind to BambL (Fig. S3) but did not mobilize the intracellular $\mathrm{Ca}^{2+}$ flux even at a very high BambL concentration (10 $\mu \mathrm{g} / \mathrm{ml})$ (Fig. 3C and D). Together, these data suggest that the surface expression of both IgD and CD19 is indispensable for the BambL-induced activation of primary mature $\mathrm{B}$ cells.

\section{CD19, IgM, and IgD are internalized upon BambL binding}

Upon binding and opening of the BCR by a cognate antigen, the receptor complex becomes internalized by endocytosis (53). Because CD19 and IgD were essential for BambL-mediated B cell activation, we tested whether BambL induced the endocytosis of these surface molecules. As another control, we also monitored the cell surface expression of IgM. After 16 hours of incubation with BambL, murine B cells expressed substantially reduced the amounts of CD19, IgD, and surprisingly also of $\operatorname{IgM}$ on the surface compared to those of unstimulated cells or B cells stimulated with anti-IgM (Fig. 4A and B). Moreover, the effects were inhibited in the presence of L-Fucose (Fig. 4A and B). The binding to and subsequent internalization of CD19, IgD, and $\operatorname{IgM}$ was specific, because other heavily glycosylated molecules, such as B220 (CD45R), showed no decrease in cell surface abundance after 16 hours of incubation with BambL (Fig. S4). Together, these observations demonstrate that BambL leads to a fucose-specific internalization of CD19, $\operatorname{IgD}$ and, to a lesser degree, also IgM.

\section{BambL induces an increase in CD86 abundance and TNF- $\alpha$ secretion}

To further elucidate the physiological effects of BambL-mediated activation, we sought to determine whether early $\mathrm{B}$ cell activation markers, such as CD86 (B7.2), were increased in response to BambL (54). CD86 is a highly glycosylated molecule and was internalized upon BambL incubation (Fig. S5A). Thus, we established a
'BambL wash-out" protocol in which B cells were first incubated with BambL at $37^{\circ} \mathrm{C}$ for $15 \mathrm{~min}$, then washed to remove unbound lectin, and left overnight to recover. We found that the increase in CD86 abundance in response to BambL was similar to that induced after activation with the TLR ligands CpG and LPS, although not to the same extent as that in response to anti-IgM $F\left(a^{\prime}\right)_{2}$ (Fig. 5A and B). The addition of L-Fucose resulted in BambL inducing only a moderate increase in CD86 cell surface expression.

To confirm that the observed effect was dependent on the presence of the BCR rather than an artifact caused by pattern-recognition receptor (PRR) stimulation, we assessed the response of BCRdeficient $\mathrm{B}$ cells to BambL. We did not observe an increase in CD86 abundance in these cells after BambL stimulation (Fig. 5C). Initial signaling events, such as $\mathrm{Ca}^{2+}$ flux, induce the differentiation of xxx cells into effector cells. For B lymphocytes, the spectrum of effector cell function includes the secretion of cytokines (55). Analyzing the supernatants of BambL-treated B cells, we detected a marked increase in tumor necrosis factor alpha (TNF $\alpha$ ) production (Fig. S5B) and secretion (Fig. 5D). TNF- $\alpha$ secretion by $\mathrm{B}$ cells is reported to be an outcome of canonical NF- $\mathrm{KB}$ signaling (56), which suggests that BambL resulted in activation of the NFKB pathway. In summary, treatment of $\mathrm{B}$ cells with BambL results in a sustained, BCRdependent increase in CD86 abundance even after its removal from these cells.

\section{BambL leads to $B$ cell death, which is partially reversible by BAFF}

Strong intracellular signaling can drive B cells into activation-induced cell death (AICD) (57-59). Because BambL binding to $\mathrm{B}$ cells resulted in the IgD- and CD19-dependent activation of intracellular signaling cascades, we tested the survival of splenic B cells after stimulation. After 24 hours of incubation with BambL, B cell viability decreased in a concentration-dependent manner (Fig. 6A). To characterize the type of BambLinduced cell death, we performed further viability assays using various inhibitors, which targeted pathways related to apoptosis, pyroptosis, and necroptosis. Pyroptosis can be induced by infection with Gram-negative bacteria (including 
Burkholderia) but has rarely been observed in $\mathrm{B}$ cells (60-62). The initiation of pyroptosis is thought to depend on caspase-1 activity (63); however, treatment of xxx cells with the Caspase- 1 inhibitor did not alter BambL-mediated cytotoxicity (fig. S6A). In contrast, the survival of BambL-treated B cells increased upon their treatment with the apoptosis inhibitor QVD, and treatment with the necroptosis inhibitor Nec-1 resulted in detectable rescue effects (Fig. S6A). Thus, B cells exposed to BambL most likely succumb to a combination of apoptosis and necroptosis.

Binding of BAFF to its receptor BAFF-R activates essential survival functions in transitional and mature B cells (29). We, therefore, tested whether BAFF could reverse or prevent BambL-induced cell death. However, co-incubation with BAFF overnight only restored the viability of BambLtreated B cells by approximately $20 \%$ (Fig. 6B). In a 4-day time course experiment with a sub-lethal concentration of BambL we observed an increase in the viability of BambL-treated $B$ cells in the presence of BAFF (Fig. S6B). This increased survival was not due to BAFF-dependent changes in BambL binding (Fig. 6C). Together, these results suggest that BambL induces B cell death, which can be partially rescued by BAFF.

\section{In vivo administration of BambL leads to splenomegaly and the loss of $B$ cells from the bone marrow}

To address the in vivo relevance of our in vitro findings, we injected BambL $(15 \mathrm{mg} / \mathrm{kg})$ i.p. into WT mice and assessed the effects of BambL at days 3 and 7 . We decided to use this concentration based on our observation that BambL is bound by soluble Igs: co-incubation with Igs neutralized BambL, reducing its binding to $\mathrm{B}$ cells and subsequently diminishing the intracellular $\mathrm{Ca}^{2+}$ release (Fig. S7).

The mice were healthy before and after injection; the application of BambL did not lead to weight loss or any other macroscopically detectable side effects. At day 3 after BambL application, the mice exhibited splenomegaly with the spleens being on average 2.7-fold heavier compared to those of PBSinjected control mice (Fig. 7A and B). On day 7, the spleen weights were statistically significantly reduced compared to those on day 3 , but were still approximately 2 -fold heavier than the spleens from the control mice (Fig. 7A and B).

The total B cell numbers in the spleens of BambLtreated mice were markedly increased, mainly due to the changes in the numbers of MFo, T1, and T2/3 populations (Fig. 7C and D; for gating strategy, see: Fig. S8A), whereas the $T$ cell numbers were not affected (Fig. S8B). The absolute cell counts of these populations had declined by day 7 (Fig. 7C and D; for gating strategy, see: Fig. S8A). MZ B cell numbers were not affected in BambL-treated mice (Fig. 7D; for gating strategy, see: Fig. S8A). Due to the successful inhibition of cell death by QVD in vitro (Fig. S6A) and its reported applicability in vivo $(64,65)$, we decided to coinject QVD with BambL into WT mice to investigate the influence of caspases on BambLmediated effects in vivo. Caspase- 6 and caspase- 8 as well as the para-caspase MALT-1 have been described to play essential roles in B cell differentiation, death, and survival $(59,66,67)$. We analyzed the BambL- and QVD-treated animals at day 3 after injection, when the full potential of the inhibitor could still be assumed. However, coapplication of QVD in vivo did not statistically significantly affect either the size of the spleens or the B cell numbers in the spleen (Fig. 7, A to D).

In the bone marrow, lymphopenia was detected on day 3 after injection of BambL, which was marked by the loss of total B cells among which the pre-, immature, and recirculation B cell subpopulations were statistically significantly reduced (Fig. 7, E and F; for gating strategy, see: fig. S8C), whereas the total numbers of bone marrow-derived cells were not changed (Fig. S8D). Similar to its effects in the spleen, QVD did not rescue the BambLinduced reduction in $\mathrm{B}$ cell numbers in the bone marrow (Fig. 7E and F). The numbers of mature recirculating B cells were statistically significantly increased by day 7 compared to day 3 after injection with BambL alone or with QVD (Fig. 7E), suggesting that the $\mathrm{B}$ cell numbers were likely restored over time. Together, these data suggest that BambL induced a transient splenomegaly and expansion of $B$ cells in the spleen with simultaneous loss of B cells from the bone marrow.

\section{BambL administration induces the accumulation of myeloid cells in the $\mathbf{x x x}$}


During the analysis of BambL-injected mice, we observed an accumulation of large and granulated cells by flow cytometric analysis (Fig. S8E). To determine the exact lineage of this population, we stained the splenocytes and bone marrow-derived cells for myeloid surface markers and calculated the absolute cell counts of myeloid populations based on a previously described gating strategy (68) and (69). On day 3 after BambL injection, we found that the amount of total $\mathrm{CD} 11 \mathrm{~b}^{+}$cells was statistically significantly increased in the spleen; specifically, neutrophils, eosinophils, macrophages, and dendritic cells (DCs) (Fig. 8A and B; for gating strategy, see: Fig. S9A). The cell numbers of these populations, with the exception of eosinophils, were reduced by day 7 (Fig. 8A and B). We further distinguished inflammatory and circulating monocyte populations, which were still increase in number on day 7 (Fig. 8C). The addition of QVD did not substantially affect the numbers of total $\mathrm{CD} 11 \mathrm{~b}^{+}$cells in BambL-treated mice. The inflammatory monocytes were statistically significantly increased in number after co-injection with QVD compared to those from mice treated only with BambL. In the bone marrow, the numbers of myeloid cells were not statistically significantly changed upon injection with BambL alone or together with QVD (Fig. 8 D to F; gating strategy: Fig. S9B).

We stained the splenic and bone marrow-derived cells with the Red Flica active polycaspases kit and Sytox viability dye to assess caspase activity. Based on a similar gating strategy as one described earlier (fig. S8, A and B), we identified the individual B cell compartments as well as the total non-B cell population. We subsequently analyzed these populations by plotting Red Flica versus Sytox, which enabled us to assess the caspase activity (Red Flica $^{+}$), to detect later stages of apoptosis (Red Flica $^{+}$Sytox $^{+}$) and to exclude necrotic cells $\left(\right.$ Sytox $\left.^{+}\right)$. We found statistically significantly increased caspase activity (Red Flica ${ }^{+}$) in splenic non-B cell populations, which was reduced after QVD application (fig. S10, A and B, left). In the bone marrow, only the pro-B cells exhibited caspase activity, wherein the Red Flica $^{+}$Sytox ${ }^{+}$ population (late apoptotic cells) was statistically significantly increased (fig. S10, C to F).
In vivo expansion of splenic $B$ cells is independent of $B C R$ signaling and of $B$ cell development in the bone marrow

To assess whether the observed in vivo phenotype after BambL injection depended on BCR signaling, we injected mice with BambL, wherein we inactivated $\mathrm{BCR}$ expression by conditionally deleting Ig $\alpha$. We used the $\operatorname{Ig} \alpha^{\mathrm{TMF}}$ mouse model (70), which we crossed with the mb1-CreER ${ }^{\mathrm{T} 2}$ mice. At day 10 after tamoxifen application, these mice had inactivated Ig $\alpha$ expression and subsequently lost BCR surface expression. We chose this mouse line for the in vivo experiments because the deletion of the Ig $\alpha$-encoding locus in these mice was more efficient (approximately 70 to $90 \%$ BCR-negative B cells) compared to the inactivation of the $\mathrm{HC}$ in the mb1-CreER ${ }^{\mathrm{T} 2} ; \mathrm{B} 1-8^{\mathrm{fl}}$ mice (approximately 15 to $20 \%$ BCR-negative B cells), which we had used in our earlier in vitro experiments. Analogously to WT mice, the mb1$\mathrm{CreER}^{\mathrm{T} 2}$; $\mathrm{Ig} \alpha^{\mathrm{TMF}}$ mice (referred to as $\operatorname{Ig} \alpha^{\mathrm{TMF}}$ mice for simplicity) were injected with $15 \mathrm{mg} / \mathrm{kg}$ BambL. On day 3 after injection, these mice exhibited splenomegaly with splenic weights increased two-fold in BambL-treated versus PBStreated animals (Fig. S11A and B). By staining the $B$ cells from the $\operatorname{Ig} \alpha^{\mathrm{TMF}}$ mice with anti-IgM and anti-IgD antibodies, we confirmed that approximately 70 to $90 \%$ of all B cells were indeed BCR-negative (Fig. S11C). We further observed an increase in the number of total B cells in the spleen, most likely due to an increased number of MFo B cells, because the transitional and $\mathrm{MZ}$ compartments were not substantially affected (fig. S11, D and E). Similar to WT mice, the T cell numbers were not changed (Fig. S8B). In the bone marrow, the total cell numbers were not markedly altered, whereas the absolute B cells counts were reduced; herein, specifically pro-, pre-, and immature B cell compartments were affected (Fig. S11F and G; for gating strategies for splenic and bone marrow cells, see fig. S11H and fig. S11I, respectively). In addition, myeloid cells in the spleen (fig. S11, J to L), as well as myeloid compartments in the bone marrow were not substantially changed (fig. S11, M to O).

To investigate the origin of the $\mathrm{B}$ cell expansion in the spleen, we blocked IL-7 receptor (IL-7R) signaling by injecting the mice with anti-IL-7R antibodies 7 days before we administered BambL, 
thereby arresting B cell development in the bone marrow. On day 3 after BambL injection (thus, day 10 after anti-IL-7R application), we observed splenomegaly (fig. S12, A and B) and increased numbers of total B cells, most likely due to an expansion of the MFo B cell compartment (fig. $\mathrm{S} 12 \mathrm{C}$ ). Whereas T1 B cell numbers in the spleen were statistically significantly increased in BambLtreated mice (fig. S12, D and E), their average numbers were 20-fold lower compared to those of BambL-treated WT mice, which had not received anti-IL-7R antibodies (Fig. 7D). Consistent with this finding, the T2/T3 population was also reduced in numbers; however, it was not further affected by BambL treatment (fig. S12, D and E).

In the bone marrow of anti-IL-7R-treated mice, the total $\mathrm{B}$ cell numbers were approximately 4.5 -fold less than those of untreated WT mice (fig. S12F and Fig. 7E). Furthermore, lymphopenia was observed after BambL administration, wherein the total B cell counts and the numbers of mature recirculating $\mathrm{B}$ cells in the bone marrow were reduced (fig. S12, F to H). The numbers of pro-, pre-, and immature B cells were markedly reduced due to anti-IL-7R treatment but were not further changed by BambL application (fig. S12, G and H). Lastly, we detected an increase in the number of neutrophils in the spleens and bone marrow of anti-IL-7R- and BambL-treated mice, whereas other myeloid lineage cells were not substantially affected (Fig. S12I to N). These results suggest that the BambL-induced accumulation of $\mathrm{B}$ cells in the spleen was most likely due to an increase in the number of MFo cells rather than a shift in lymphopoiesis from the bone marrow to the spleen. Furthermore, the lack of the mature recirculating population in the bone marrow demonstrates that mature B cells did not circulate but remained in the spleen.

\section{BambL is localized in the cytosol of B. ambifaria} The cellular localization of BambL in B. ambifaria has not yet been investigated. We therefore assessed the subcellular localization of BambL in $B$. ambifaria biofilms on agar plates and found that the lectin was not released but was exclusively retained in the cytosol of the bacteria (fig. S13).

\section{Discussion}

Pathogens express adhesins and toxins to aid in infection and the colonization of host tissues. Glycan-binding factors, such as the influenza A hemagglutinin, were identified by their ability to aggregate red blood cells $(71,72)$, whereas toxins, such as Shiga toxin, were shown to use glycolipids on epithelial cells as docking sites for entry $(11,73)$. Here, we provide insights into the interaction between fucose-binding bacterial lectins and B cells and demonstrate that the lectins LecB and BambL trigger BCR-dependent activation in vitro, ultimately resulting in activation-induced death of $\mathrm{B}$ cells. This signaling cascade depended mainly on CD19 and IgD, because CD19- and IgD-deficient B cells failed to mobilize intracellular $\mathrm{Ca}^{2+}$ upon exposure to BambL. To a lesser extent, BambL also bound to IgM, as seen in the experiments using high lectin concentrations, wherein preference for IgD was lost. Note that studies on monovalent versus multivalent $\mathrm{BCR}$ antigens suggest that a strong burst of $\mathrm{Ca}^{2+}$ release only takes place after stimulation with soluble multivalent antigens (30, $74,75)$. Interesting in this context is the finding that the hinge region of $\operatorname{IgD}$ is more flexible compared to the more rigid IgM-BCR, rendering the $\operatorname{IgD}$ molecule better suited to bind to multivalent antigens $(30,33)$. Consistent with our experiments are reports showing that BCR signaling upon multivalent antigen triggering relies to a large extent on the function of IgD and CD19 and less on $\operatorname{IgM}$ (33). Whereas antigen-binding involves the $\mathrm{CDR}$ loops of the variable domains of the $\mathrm{HC}$ and LC, BambL most likely binds to glycan residues at the constant and hinge regions of the BCR, indicating a difference in mechanism, despite similarities in target receptor affinities. In addition, the signaling of the chemokine receptor CXCR4 engages BCR crosstalk by activating the actin cytoskeleton and also depends on IgD and CD19 expression (76). Both IgM and IgD possess five Nglycosylation sites at each constant region, which differ in their positions. The influence of carbohydrates on the regulation and activation of the BCR is not well understood at present. However, a study by Chen et al. demonstrated that the glycoproteins that surround IgD- and IgMBCRs strongly differ and that this might explain their divergent signaling behaviors (77). BambLinduced activation resembles that induce by $\mathrm{B}$ cell superantigens, such as the microbial toxins Staphylococcus protein A or Peptostreptococcus 
protein $\mathrm{L}$, which bind to conserved regions of the BCR by protein-protein interactions $(78,79)$, but are glycosylation-independent.

In addition to $\mathrm{Ca}^{2+}$ release, short-term stimulation of $\mathrm{B}$ cells with BambL initiated increased cell surface CD86 expression and the activation of effector functions, such as the secretion of TNF- $\alpha$. In canonical $B$ cell activation, the surface expression of CD86 is increased to enable T cell costimulation by $\mathrm{CD} 28$. We found that long-term stimulation with BambL overnight again decreased CD86 cell surface expression and caused cell death. The loss of surface CD86 might be explained by a direct interaction with BambL: if CD86 is fucosylated, it will likely bind to BambL after prolonged exposure with the lectin, which could lead to its internalization.

After initial B cell activation, BambL induced B cell death, whereas co-treatment with soluble BAFF, which activates pro-survival signals of the noncanonical NFKB and PI3K pathways, partially protected the lectin-treated cells $(28,80,81)$. Additionally, the poly-caspase inhibitor QVD, which blocks caspases-1,-3,-8,-9, and -12 , the major players during apoptosis (82), and the necroptosis inhibitor Nec-1 rescued B cells from BambL-induced cell death in vitro.

To address the role of BambL in vivo, we injected BambL i.p. into WT and Ig $\alpha^{\mathrm{TMF}}$ mice. We observed a transient increase in B cell numbers in the spleen, as well as splenomegaly in WT and Ig $\alpha^{\mathrm{TMF}}$ mice, which lack the surface BCR, suggesting that the effect of BambL on B cells in vivo was independent of BCR signaling but might result from stimulation of other B cell surface receptors (Fig. 7 and fig. S11). This phenotype is reminiscent of reports ascribing splenomegaly to various infections, injections with BCR-targeting antibodies, and the application of excess BAFF (83-85). The increase in splenic B cell numbers was accompanied by a loss of B cells in the bone marrow (Fig. 7). To reconcile the lymphopenia in the bone marrow with the simultaneous accumulation of B cells in the spleen three days after BambL application, we first assumed that BambL triggered an activationinduced migration of $\mathrm{B}$ cells from the bone marrow to the spleen. However, blocking B lymphopoiesis in the bone marrow with anti-IL-7R antibodies revealed that the accumulation of mature B cells most likely occurred directly in the spleen due to the hyper-proliferation of MFo cells (Fig. S12). The loss of $\mathrm{B}$ cells in the bone marrow was likely due to BambL-induced enhanced apoptosis at the pro-B cell stage (Fig. S10). Note that the B cell populations in the bone marrow were in the process of recovering, and the spleen sizes and weights, as well as splenic B cell numbers, decreased to almost normal levels by day 7 (Fig. 7). This is to some extent consistent with our in vitro observation that BambL triggered the activation-induced death of B cells (Fig. 6). However, although we did not detect enhanced apoptosis of splenic B cells in vivo, we cannot exclude the possibility that BambL induced other cell death mechanisms. A study identified a previously uncharacterized proliferation-limiting mechanism: a DNA damage response-induced cell cycle arrest upon the initial hyperproliferation of EBV- or CpG-stimulated B cells. This cell cycle arrest mechanism inhibits proliferation without inducing apoptosis (86). Another study demonstrated that B cells transiently activated with antigen return to a quiescent state in the absence of $\mathrm{T}$ cell help (87). It is feasible that similar mechanisms control the initial hyper-proliferation and subsequent proliferation arrest upon treatment with BambL, particularly because we did not observe any changes in the numbers of $\mathrm{T}$ cell populations after BambL injection, indicating a lack of enhanced $\mathrm{T}$ cell activity (Fig. S8B).

We also observed a transient accumulation of myeloid lineage cells in the spleens of BambLtreated WT mice, including neutrophils, eosinophils, macrophages, and DCs (Fig. 8). This indicates that BambL not only targets B cells in vivo, but also other cell types carrying fucosebearing receptors. It is likely that the temporary increase in the numbers of these cells further contributes to splenomegaly. In addition, myeloid lineage cells produce B cell stimulatory factors, including BAFF $(88,89)$. Because BAFF dampened BambL-induced $\mathrm{B}$ cell death in vitro (Fig. 6), increased amounts of BAFF, secreted by activated myeloid cells, might account for the BambL-mediated proliferation of $B$ cells and protection from BambL-induced $\mathrm{B}$ cell death in vivo. Note that because caspase activity was enhanced in splenic non-B cell populations, we assume that BambL induced a temporary increase 
in the number of myeloid cells with subsequent apoptosis, thereby reducing their numbers until day 7 (Fig. S10). Because we partially reversed BambLinduced B cell death with the polycaspase-inhibitor $Q V D$ in vitro, we co-injected this inhibitor into the BambL-treated mice but observed no substantial effect. The activity of caspases has been described to play a role not only in the induction of cell death, but also during the differentiation of immune cells (82). In our experimental set-up, we only detected caspase activity in non-B cells and pro-B cells.

\section{B. ambifaria is a member of the Burkholderia} cepacia complex (Bcc). Burkholderia species comprising the $\mathrm{Bcc}$ are often found in $\mathrm{CF}$ patients and they release multiple lectins, which have not been investigated to date $(4,90)$. It is likely that these bacteria share common features. For example, BambL from Burkholderia ambifaria shares a high protein sequence similarity with other lectins from B. cepacia $(99 \%), B$. pyroccinia $(95 \%)$, and $B$. contaminans (94\%) (according to an uniprot-based BLAST analysis). The expression dynamics and cellular localization of BambL in B. ambifaria are poorly studied to date. Previous studies reported that LecB from the B. ambifaria-related bacterium $P$. aeruginosa was located in the outer surface of the bacterial cell wall $(10,91)$. We found BambL exclusively in the cytosol of B. ambifaria extracts. This finding is not surprising because other pathogenic bacteria withhold their soluble lectins; for example, the lectin Shiga toxin-B subunit is limited to the cytoplasm and periplasm (92) and is released by a triggered, phage-mediated bacterial lysis (11, 93, 94). Similarly, B. ambifaria may release BambL in response to specific environmental changes that are yet to be discovered.

Another study has correlated the expression of LecA and LecB from $P$. aeruginosa with its pathogenicity, wherein bacterial cytotoxicity and adhesion were reduced in mutant LecA and LecB strains in vitro and the bacterial burden and dissemination of the mutant strains were decreased in vivo (95). Moreover, our group previously reported that the invasiveness of $P$. aeruginosa depended directly on the expression of LecA (12). The role of BambL in infection in general and in $\mathrm{CF}$ in particular remains elusive and the site of $\mathrm{B}$ cell encounter with BambL in vivo is not known to date.
The lymph nodes may not be the only sites where B cells encounter their antigens. Direct interaction of $\mathrm{B}$ cells with bacterial proteins in the periphery has been previously described $(96,97)$. For example, Chen et al. demonstrated the existence, in the mucosa of the upper respiratory tract, B cells bearing $\mathrm{IgD}$ alone that become class-switched from IgM in a non-canonical manner of B cell maturation (98). It is possible that the binding of BambL to soluble fucosylated proteins, such as antibodies, may dampen its effectiveness in vivo. However, a study demonstrated that circulating IgG molecules do not inhibit the function of Staphylococcus aureas superantigen $\mathrm{SpA}$ in vivo but rather potentiate its effects (99). We are aware that SpA binding to $\mathrm{B}$ cells is glycosylation-independent; therefore, we cannot directly compare it to the binding of BambL to fucosylated epitopes. However, we cannot exclude the possibility that similar mechanisms occur during an infection with B. ambifaria or after injection with purified BambL.

It is important to emphasize that all of the observed changes in cellular physiology are likely due to the ability of the lectin to bind to fucosylated host cell receptors. Although BambL may bind to various targets in vivo, our observations suggest that the effect on mature BCR signaling through its glycosylated structures is underestimated. For the murine pre-BCR, core-fucosylation has been reported to be indispensable for functional $\mathrm{HC}$ expression (100). The increase in fucosylation on epithelial cells protects against many intestinal bacterial infections (101) but increases the susceptibility to other (for example, viral) pathogens (102). However, a direct link between fucosylation and host-pathogen signaling has been difficult to prove, partly due to the strong polymorphism of fucosyltransferases (103). Fucosylated epitopes have been identified as functional receptors for the B-subunit of cholera toxin, which binds specifically to monosialotetrahexosylganglioside (GM1) and is often used as a lipid raft marker (13). Furthermore, a viral lectin, the hemagglutinin of influenza $A$ virus, has been classified as a pan-B cell activator through its binding to the sialic acid residues of a reconstituted BCR (104), resulting in the hypothesis that this carbohydrate-dependent and superantigen-like B cell activation mechanism might be evolutionarily conserved; however, 
bacterial lectins have not been included in the list of typical danger- or pathogen-associated molecular patterns (DAMPs or PAMPs) so far.

We propose a mechanism of $\mathrm{B}$ cell activation by bacteria. In vitro, BambL specifically binds to the fucose residues of BCRs. This interaction initiates diverse, crucial B cell signaling processes, which depend on the presence of the surface receptors IgD and CD19, and eventually cause B cell death. In vivo, BambL application caused a rapid cellular immune response, which involved not only B cells, but also innate immune cells, and subsided within a week. This might pose a risk to immunocompromised patients unable to mount an effective response to pathogenic particles. Whether our finding can be translated to a natural infection remains to be elucidated. However, this study opens up the possibility for further in-depth research of host-pathogen interactions and reveals that highly regulated mechanisms of $\mathrm{B}$ cell signaling and complex BCR expression programs can be hijacked by glycan-targeting bacterial lectins.

\section{Materials and Methods \\ Production of recombinant bacterial lectins}

BambL (uniprot-ID: Q0B4G1_BURCM) and LecB (uniprot-ID: Q9HYN5 PSEAE) were expressed in $E$. coli and purified by affinity chromatography, as previously described $(8,9)$. The freeze-dried powder was dissolved in phosphate-buffered saline (PBS) without $\mathrm{Ca}^{2+}$ or $\mathrm{Mg}^{2+}$ at $1 \mathrm{mg} / \mathrm{ml}$. To avoid contamination by LPS, the lectins were further purified on endotoxin removal traps (hyglos), sterile-handled, and regularly checked for LPS contamination with the Pierce LAL chromogenic assay (Thermo Scientific). Activation experiments (measurement of $\mathrm{Ca}^{2+}$ release and increased CD86 expression) included additional controls with boiled BambL $\left(15 \mathrm{~min}\right.$ at $\left.95^{\circ} \mathrm{C}\right)$ to exclude possible effects of heat-stable LPS contaminants originating from the recombinant protein production. For fluorescent labeling, the purified lectins were covalently tagged with the Alexa Fluor 488 (Thermo Scientific), Cy-3, or Cy-5 (GE Healthcare) labeling kits according to the manufacturers' instructions and repurified on a Pierce ZebaSpin DSalt polyacrylamide desalting column (Thermo Scientific).

\section{Mice}

All animal experiments were conducted with mice aged 8 to 12 weeks, were performed in accordance with the German Animal Welfare Act, and were reviewed by the regional council and approved under license $\# \mathrm{G}-09 / 103$. Mice were bred in the animal facility of the Max Planck Institute of Immunology and Epigenetics (Freiburg, Germany) and the University of Ulm (Ulm, Germany). For inducible, B cell-specific deletion, mice were orally administered tamoxifen citrate (Ratiopharm) dissolved in 20\% ClinOleic (Baxter) as previously described (80). The course of tamoxifen treatments and the interval between doses were pre-optimized for maximal recovery of the deleted cells and are outlined below (81). In this study, the following mice strains were used: $\mathrm{C} 57 \mathrm{BL} / 6$ WT or heterozygous $\mathrm{mEGFP} / \mathrm{mb}^{\text {inv }}$ (105) $\left(\mathrm{GFP}^{+} \mathrm{B}\right.$ cells); $\mathrm{B}$ cells from $\operatorname{IgM}^{-/-}$(47) and $\mathrm{IgD}^{-/-}$mice were also used (106). CD19 ${ }^{-/-}$B cells were obtained from homozygous CD19-CreER ${ }^{\mathrm{T} 2}$ mice (kindly provided by Prof. Dr. Marc Schmidt-Supprian) $(81,107)$. To generate CD19/IgD double-deficient B cells, homozygous CD19-CreER ${ }^{\mathrm{T} 2}$ mice were crossed with homozygous $\mathrm{IgD}^{-/-}$mice and then intercrossed. $\mathrm{HC}^{-/-} \mathrm{B}$ cells were generated by crossing $\mathrm{HC}^{\mathrm{B} 1-8 \mathrm{fl} / \mathrm{JhT}}$ mice (44) with mb1CreER ${ }^{\mathrm{T} 2}$ mice and were analyzed 7 days after tamoxifen application. For the BambL injection experiments, $\operatorname{Ig} \alpha^{\mathrm{TMF}}$ mice (70) were crossed with mb1-CreER ${ }^{\mathrm{T} 2}$ mice; their offspring are termed $\operatorname{Ig} \alpha^{\mathrm{TMF}}$ mice in this manuscript for simplicity. The mice were injected with BambL at day 7 after tamoxifen treatment and cells were extracted and analyzed 3 days later. $\operatorname{Ig} \alpha^{-/-}$B cells were generated by crossing $\mathrm{mb} 1 / \mathrm{mEGFP}^{\text {inv }}$ (105) mice with mb1-CreER ${ }^{\mathrm{T} 2}$ mice $(45,81)$ and were obtained on day 10 after tamoxifen treatment. Furthermore, we used $\mathrm{Lyn}^{-/}$ mice (108), mice with B cell-specific conditional Syk deletion $\left(\mathrm{Syk}^{--}\right)$(81), and Blimp ${ }^{\mathrm{GFP}}$ mice (49). The animals were sacrificed using $\mathrm{CO}_{2}$, and the spleens and bone marrow were extracted under nonsterile conditions. B cells were then purified by magnetic or flow cytometry-based separation.

\section{Isolation of murine B cells by magnetic-activated cell sorting (MACS) depletion and flow cytometry-based sorting}

Cell separation was performed by MACS depletion or flow cytometry-based sorting: spleen-derived B cells were obtained by MACS-based negative selection using the B cell isolation kit (Miltenyi 
Biotech) according to the manufacturer's instructions. The cells were then separated using an AutoMACS (Miltenyi Biotech). Spleen-derived B cells from heterozygous $\mathrm{mEGFP} / \mathrm{mb} 1^{\text {inv }}$ mice as well as $\mathrm{GFP}^{+} \mathrm{B}$ cells from $\mathrm{Ig}^{-/-}$mice were separated by FACS based on their abundance of GFP with a FACSAria (BD) cell sorter. HCdeficient $B$ cells were sorted based on the lack of $\operatorname{IgM}$ and $\mathrm{IgD}$ expression, as described elsewhere (45). After separation, B cells were analyzed for purity and viability with an anti-CD19 antibody (6D5, BioLegend) and 7-AAD (BioLegend) or a fixable viability dye (eBioscience).

\section{Flow cytometry analysis}

Extracellular staining for flow cytometry (FACS) analysis was performed with 0.5 to $1 \times 10^{6}$ cells $/ \mathrm{ml}$ in FACS buffer (PBS; 3\% FCS, $0.05 \% \mathrm{NaN}_{3}$ ) on ice for $20 \mathrm{~min}$. Before staining, the cells were incubated with the anti-Fc receptor-blocking antibody (CD16/32, Clone 2.4G2; own production) on ice for at least $5 \mathrm{~min}$. For the extracellular staining of $\mathrm{B}$ cells, the following antibodies were used: anti-CD19 Alexa Fluor647 or PE (eBio1D3; eBioscience and 6D5; BioLegend), anti-IgD FITC or PE (11-26c.2a; BioLegend), anti-CD23 Alexa Fluor647 or PE-Cy7 (B3B4; BioLegend or 2G8; Southern Biotech), anti-CD24 (M1/69; Biolegend,), anti-CD25 (PC61.5, eBioscience), anti-BAFF-R biotin (polyclonal; R\&D Systems), anti-IgM eFluor450 (eB121-15F9), anti-CD93 PE or APC (AA4.1), anti-CD86 PE (GL1) (all eBioscience). For the myeloid stainings, we used anti-CD11c (N418, Biolegend), anti-F4/80 (BM8, eBiosciences), anti-CD11b (M1/70; eBioscience), anti-Ly6C (HK1.4; eBioscience), anti- CD3 $\varepsilon$ (145$2 \mathrm{C} 11$, eBioscience). The cells were washed with $700 \mu \mathrm{l}$ of FACS buffer to remove the residual antibodies and centrifuged at $300 \times \mathrm{g}$ at $4{ }^{\circ} \mathrm{C}$ for $5 \mathrm{~min}$. All antibodies used in this study were tested for lectin crossreactivity before staining. Cyan ADP (Beckman Coulter), LSRII, and LSRFortessa (both Becton Dickinson) cell analyzers were used for data acquisition. For intracellular staining, cells were fixed and permeabilized using the Fix\&Perm Kit (ADG Bio Research) and stained with anti-TNF $\alpha$ antibody (clone: MP6-XT22, eBioscience) dissolved in saponin-based buffer. Cytometry data were analyzed with Flow Jo software (Tree Star).

\section{Caspase-activity assay}

Cells were stained with the SR-FLICA Caspase Assays kit (ImmunoChemistry Technologies) according to the manufacturer's instructions. In brief, the cells were isolated, stained with detection reagent for 1 hour at room temperature, and washed with the specific apoptosis buffer containing PBS, which was provided with the kit.

\section{$\mathrm{Ca}^{2+}$ analysis and binding assays}

$\mathrm{Ca}^{2+}$ flux in purified mature B cells was measured by flow cytometry using the cell-permeable fluorescent dye Indo-1 (Life technologies) cells loaded as described previously (45). The following stimuli were used: anti-IgM $\mathrm{F}\left(\mathrm{ab}^{\prime}\right)_{2}$ (goat polyclonal; Jackson Immunoresearch), anti-kappa $\mathrm{F}\left(\mathrm{ab}^{\prime}\right)_{2}$ and anti-IgD $\mathrm{F}\left(\mathrm{ab}^{\prime}\right)_{2}$ (both polyclonal; Southern Biotech). The lectins were applied in different concentrations ranging from $250 \mathrm{ng} / \mathrm{ml}$ to $10 \mu \mathrm{g} / \mathrm{ml}$, diluted in culture medium containing $1 \%$ FCS. Simultaneous to the detection of $\mathrm{Ca}^{2+}$ release, the binding of BambL-Cy5 in the corresponding concentration was assessed over time. Intracellular $\mathrm{Ca}^{2+}$ flux and extracellular binding were measured on an LSRII flow cytometer (Becton Dickinson).

\section{Lectin-dependent increase in CD86 cell surface abundance}

B cells were seeded at $1 \times 10^{6}$ cells $/ \mathrm{ml}$ in complete medium and stimulated in a 96-well U-bottom plate with the lectin dissolved in complete medium. For the binding assays, B cells were stimulated on ice for $15 \mathrm{~min}$ and then analyzed for lectin binding by flow cytometry. For the "wash out" procedure, cells were stimulated with $\mathrm{BambL}$ at $37^{\circ} \mathrm{C}$ for $15 \mathrm{~min}$, which was followed by two washing steps with $2 \mathrm{ml}$ of PBS. As a positive control, we used polyclonal anti-IgM $\mathrm{F}(\mathrm{ab}) 2$ (goat polyclonal; Jackson Immunoresearch, $10 \mu \mathrm{g} / \mathrm{ml})$; unmethylated $\mathrm{CpG}$ oligonucleotides (Invivogen; ODN 1826, $2.5 \mu \mathrm{M}$ ), and LPS (Sigma; $10 \mu \mathrm{g} / \mathrm{ml}$ ). The cells were harvested $16 \mathrm{~h}$ after stimulation and the cell surface abundnace of the activation marker CD86 was analyzed by flow cytometry using anti-CD 86 (clone: GL1; eBioscience).

\section{Measurement of TNF- $\alpha$ by ELISA}

The concentration of secreted TNF-a was measured by enzyme-linked immunosorbent assay (ELISA). The extracted splenic B cells were treated with BambL overnight and the cell culture medium was collected by centrifugation and subsequent 
filtration. The amount of TNF- $\alpha$ released was determined using the anti-mouse TNF $\alpha$ Ready-SetGo ELISA kit (eBioscience) according to the manufacturer's instructions.

\section{Survival assays}

Primary, spleen-derived murine $\mathrm{B}$ cells were purified and cultured at $37^{\circ} \mathrm{C}$ and $7.5 \% \mathrm{CO}_{2}$ in complete medium, Iscove's MDM (Biochrom, Merck) supplemented with 10\% heat-inactivated FCS, $\quad 1 \%$ Gibco Penicillin-Streptromycin (100 units $/ \mathrm{ml}$ penicillin, $100 \mu \mathrm{g} / \mathrm{ml}$ streptomycin) and $2 \mathrm{mM}$ Gibco L-glutamine (all Thermo Scientific). The cells were either incubated in medium alone or in the presence of $100 \mathrm{ng} / \mathrm{ml}$ human recombinant BAFF (hrBAFF, ImmunoTools) over a period of 4 days. The medium was exchanged every second day and supplemented with fresh hrBAFF. Cell viability was assessed with a fixable viability dye (eBioscience). Annexin V and Propidium Iodide staining was not applicable for the survival assays, because of reported false positivity by catfish egg lectin (109). For the viability analysis, the percentages were obtained from the ungated, viability dye-negative (healthy) cells. The following inhibitors were first titrated on B cells and then used in this study: necrostatin (Nec-1), poly-Caspase inhibitor QVD (Q-VD-PH), and caspase-1 inhibitor Z-WEHD-FMK (all R\&D Biosystems).

\section{Intraperitoneal injection of BambL}

Expecting that a major fraction of BambL would be intercepted by soluble Igs and carbohydrates expressed on erythrocytes, we decided to inject BambL in PBS (without adjuvants) intraperitoneally instead of intravenously. In a dose-dependency study, we determined the concentration of $15 \mathrm{mg} / \mathrm{kg}$ to be comparable to the doses of BCR-targeting antibodies that had been used previously (84). Control mice received the same volume of PBS i.p.. Seven days after injection, we extracted the spleens and bone marrow to further analyze the cells by flow cytometry. Experimental mice did not suffer at any point of the i.p. treatment experiments and did not lose any body weight during the study. QVD was co-applied at $20 \mathrm{mg} / \mathrm{kg}$, as suggested by the manufacturer (R\&D Biosystems).
BambL localization in Burkholderia ambifaria

Bacteria were fractionated based on the LecB localization protocol for Pseudomonas aeruginosa published by Tielker et al (10). B. ambifaria strain HSJ1 was grown as a biofilm on agar plates $(30 \mathrm{~g} / 1$ tryptic soy broth, $15 \mathrm{~g} / 1$ agar, Carl Roth) for 72 hours. Bacteria were detached and collected in $1 \mathrm{ml}$ of $140 \mathrm{mM} \mathrm{NaCl}$, which yielded an optical density of $\sim 160$ at $600 \mathrm{~nm}$. A sample $(100 \mu \mathrm{l})$ of this suspension was subjected to fractionation, whereas another $100-\mu 1$ sample was used to prepare whole-cell lysates (WC). Bacteria were collected by centrifugation $(5 \mathrm{~min}$ at $3,000 \times \mathrm{g})$ and the supernatant was sterile-filtered before the BambL concentration in the extracellular space (E) was determined. The bacterial outer membrane was lysed for $30 \mathrm{~min}$ at room temperature in $500 \mu \mathrm{l}$ of $100 \mathrm{mM}$ Tris/ $\mathrm{HCl}(\mathrm{pH} \mathrm{8})$ containing $20 \%(\mathrm{w} / \mathrm{v})$ sucrose, $2.5 \mathrm{mM}$ EDTA, and $20 \mu \mathrm{g}$ lysozyme (Sigma-Aldrich). Spheroblasts were collected by centrifugation (20 min at $10,000 \mathrm{xg})$; the supernatant served as the periplasmic $(\mathrm{P})$ fraction. The spheroblasts were sonicated (Bandelin Sonopuls mini20, probe MS 2.5) in $500 \mu \mathrm{l}$ of $100 \mathrm{mM}$ Tris/ $\mathrm{HCl}(\mathrm{pH} 8)$ and undisrupted cells were separated by centrifugation $(5 \mathrm{~min}$ at $5,000 \times \mathrm{g})$. The supernatant was centrifuged at $20,000 \times \mathrm{g}$ for $45 \mathrm{~min}$ to collect the membrane (M) fraction; the resulting supernatant was used as the cytoplasmic (C) fraction. For Western blotting analysis, equivalent volumes of each cellular fraction were used to directly compare the relative amounts of BambL. Recombinantly produced BambL was loaded as a positive control. Samples were boiled with SDS-PAGE sample buffer and separated in an $18 \%$ polyacrylamide gel, followed by electrophoretic transfer to a nitrocellulose membrane. After transfer, proteins were stained reversibly with Ponceau S solution (SigmaAldrich) and photographed. BambL was detected with a polyclonal antibody raised against its $\mathrm{C}$ terminus (Eurogentec) and an anti-rabbit IgG-HRP conjugate using the Clarity ECL kit (Bio-Rad).

\section{Software and statistical analysis}

Adobe Illustrator CC 2017 (Adobe) and Prism7 (Graphpad) software were used for figure preparation and statistical analysis, respectively. The statistical significance of the observed differences was evaluated by an unpaired, two- 
tailed Student's $t$-test (if not indicated otherwise). $* P<0.05, * * P<0.01, * * * P<0.001$; ns, not significant. In the in vivo experiments, the exact $P$ values are indicated; differences that were not statistically significantly different are not depicted.

\section{Supplementary Materials}

Fig. S1. Lectin binding and gating of $\mathrm{HC}^{-/-} \mathrm{B}$ cells. Fig. S2. Gating schemes and IgD surface expression of specific B cell populations.

Fig. S3. Characterization of CD19-, IgM-, and IgDdeficient B cells.

Fig. S4. B220 is not internalized upon BambL binding.

Fig. S5. Internalization of CD86 and intracellular increase in TNF- $\alpha$ in BambL-treated primary B cells.

Fig. S6. Stimulation of cells with BambL in the presence of cell death inhibitors.

Fig. S7. BambL binds to soluble immunoglobulins. Fig. S8. Analysis of lymphoid lineage cells after in vivo injection of BambL.

Fig. S9. Analysis of myeloid lineage cells after in vivo injection of BambL.

Fig. S10. Non-B cells in the spleen show an increase in caspase activity upon BambL injection.

Fig. S11. BambL injection leads to splenomegaly and $\mathrm{B}$ cell accumulation in $\mathrm{Ig} \alpha^{\mathrm{TMF}}$ mice.

Fig. S12. Mice treated with anti-IL-7R antibody exhibit splenomegaly and B cell proliferation in the spleen.

Fig. S13. B. ambifaria biofilms do not secrete BambL but retain it in the cytosol. 


\section{References and Notes}

1. T. Coenye, E. Mahenthiralingam, D. Henry, J. J. LiPuma, S. Laevens, M. Gillis, D. P. Speert, P. Vandamme, Burkholderia ambifaria sp. nov., a novel member of the Burkholderia cepacia complex including biocontrol and cystic fibrosis-related isolates, Int. J. Syst. Evol. Microbiol. 51, 1481-1490 (2001).

2. P. D. Lister, D. J. Wolter, N. D. Hanson, Antibacterial-Resistant Pseudomonas aeruginosa: Clinical Impact and Complex Regulation of Chromosomally Encoded Resistance Mechanisms, Clinical Microbiology Reviews 22, 582-610 (2009).

3. T. Coenye, P. Vandamme, Diversity and significance of Burkholderia species occupying diverse ecological niches, Env. Microbiology 5, 719-729 (2003).

4. M. S. Saldias, M. A. Valvano, Interactions of Burkholderia cenocepacia and other Burkholderia cepacia complex bacteria with epithelial and phagocytic cells, Microbiology 155, 2809-2817 (2009).

5. K. A. Ramsay, C. A. Butler, S. Paynter, R. S. Ware, T. J. Kidd, C. E. Wainwright, S. C. Bell, Factors Influencing Acquisition of Burkholderia cepacia Complex Organisms in Patients with Cystic Fibrosis, Journal of Clinical Microbiology 51, 3975-3980 (2013).

6. G. Pradenas, B. Ross, A. Torres, Burkholderia cepacia Complex Vaccines: Where Do We Go from here? Vaccines 4, 10-14 (2016).

7. E. C. Nannini, A. Ponessa, R. Muratori, P. Marchiaro, V. Ballerini, L. Flynn, A. S. Limansky, Polyclonal outbreak of bacteremia caused by Burkholderia cepacia complex and the presumptive role of ultrasound gel, Brazilian Journal of Infectious Diseases 19, 543-545 (2015).

8. A. Imberty, M. Wimmerova, E. P. Mitchell, N. Gilboa-Garber, Structures of the lectins from Pseudomonas aeruginosa: insights into the molecular basis for host glycan recognition, Microbes and Infection 6, 221-228 (2004).

9. A. Audfray, J. Claudinon, S. Abounit, N. Ruvoen-Clouet, G. Larson, D. F. Smith, M. Wimmerova, J. Le Pendu, W. Römer, A. Varrot, A. Imberty, Fucose-binding Lectin from Opportunistic Pathogen Burkholderia ambifaria Binds to Both Plant and Human Oligosaccharidic Epitopes, Journal of Biological Chemistry 287, 4335-4347 (2012).

10. D. Tielker, S. Hacker, R. Loris, M. Strathmann, J. Wingender, S. Wilhelm, F. Rosenau, K.-E. Jaeger, Pseudomonas aeruginosa lectin LecB is located in the outer membrane and is involved in biofilm formation, Microbiology 151, 1313-1323 (2005).

11. L. Johannes, W. Römer, Shiga toxins - from cell biology to biomedical applications, Nature Reviews Microbiology 8, 105-116 (2009).

12. T. Eierhoff, B. Bastian, R. Thuenauer, J. Madl, A. Audfray, S. Aigal, S. Juillot, G. E. Rydell, S. Muller, S. de Bentzmann, A. Imberty, C. Fleck, W. Römer, A lipid zipper triggers bacterial invasion, PNAS 111, 12895 12900 (2014).

13. A. M. Wands, A. Fujita, J. E. McCombs, J. Cervin, B. Dedic, A. C. Rodriguez, N. Nischan, M. R. Bond, M. Mettlen, D. C. Trudgian, A. Lemoff, M. Quiding-Järbrink, B. Gustavsson, C. Steentoft, H. Clausen, H. Mirzaei, S. Teneberg, U. Yrlid, J. J. Kohler, Fucosylation and protein glycosylation create functional receptors for cholera toxin, eLife 4, 593-78 (2015).

14. C. Cott, R. Thuenauer, A. Landi, K. Kühn, S. Juillot, A. Imberty, J. Madl, T. Eierhoff, W. Römer, Pseudomonas aeruginosa lectin LecB inhibits tissue repair processes by triggering $\beta$-catenin degradation, $B B A$ - Molecular Cell Research 1863, 1106-1118 (2016).

15. D. Avichezer, N. Gilboa-Garber, PA-II, the L-fucose and D-mannose binding lectin of Pseudomonas aeruginosa stimulates human peripheral lymphocytes and murine splenocytes, FEBS Letters 216, 62-66 (1987).

16. N. Sharon, History of lectins: from hemagglutinins to biological recognition molecules, Glycobiology 14, 53R-62R (2004).

17. R. Übelhart, M. P. Bach, C. Eschbach, T. Wossning, M. Reth, H. Jumaa, N-linked glycosylation selectively regulates autonomous precursor BCR function, Nature Immunology 11, 759-765 (2010). 
18. J. Haimovich, N. Ben Moshe, Y. Raviv, N. Hollander, All oligosaccharide moieties of the mu chains in the pre-BCR are of the high-mannose type, Molecular Immunology 48, 351-355 (2010).

19. D. Schneider, M. Dühren-von Minden, A. Alkhatib, C. Setz, C. A. M. van Bergen, M. Benkißer-Petersen, I. Wilhelm, S. Villringer, S. Krysov, G. Packham, K. Zirlik, W. Römer, C. Buske, F. K. Stevenson, H. Veelken, H. Jumaa, Lectins from opportunistic bacteria interact with acquired variable-region glycans of surface immunoglobulin in follicular lymphoma, Blood 125, 3287-3296 (2015).

20. W. G. Kerr, L. M. Hendershot, P. D. Burrows, Regulation of IgM and IgD expression in human B-lineage cells, J. Immunol. 146, 3314-3321 (1991).

21. K. Rajewsky, Clonal selection and learning in the antibody system, Nature 381, 751-758 (1996).

22. R. Geisberger, M. Lamers, G. Achatz, The riddle of the dual expression of IgM and IgD, Immunology, 429-437 (2006).

23. J. M. Hombach, T. Tsubata, L. Leclercq, H. Stappert, M. G. Reth, Molecular Components of the IgM Antigen Receptor Complex, J. Immunol., 659-662 (1989).

24. A. Weiss, D. R. Littman, Signal transduction by lymphocyte antigen receptors, Cell 76, 263-274 (1994).

25. J. C. Cambier, C. M. Pleiman, M. R. Clark, Signal transduction by the B cell antigen receptor and its coreceptors, Annu. Rev. Immunol. 12, 457-486 (1994).

26. R. A. Barrington, M. Zhang, X. Zhong, H. Jonsson, N. Holodick, A. Cherukuri, S. K. Pierce, T. L. Rothstein, M. C. Carroll, CD21/CD19 Coreceptor Signaling Promotes B Cell Survival during Primary Immune Responses, J. Immunol. 175, 2859-2867 (2005).

27. D. A. Tuveson, R. H. Carter, S. P. Soltoff, D. T. Fearon, CD19 of B cells as a surrogate kinase insert region to bind phosphatidylinositol 3-kinase, Science 260, 986-989 (1993).

28. P. Schneider, F. Mackay, V. Steiner, K. Hofmann, J. L. Bodmer, N. Holler, C. Ambrose, P. Lawton, S. Bixler, H. Acha-Orbea, D. Valmori, P. Romero, C. Werner-Favre, R. H. Zubler, J. L. Browning, J. Tschopp, BAFF, a novel ligand of the tumor necrosis factor family, stimulates B cell growth, J Exp Med 189, 17471756 (1999).

29. A. Craxton, K. E. Draves, A. Gruppi, E. A. Clark, BAFF regulates B cell survival by downregulating the BH3-only family member Bim via the ERK pathway, J Exp Med 202, 1363-1374 (2005).

30. C. Volkmann, N. Brings, M. Becker, E. Hobeika, J. Yang, M. Reth, Molecular requirements of the B-cell antigen receptor for sensing monovalent antigens, EMBO J. 35, 2371-2381 (2016).

31. F. D. Batista, D. Iber, M. S. Neuberger, B cells acquire antigen from target cells after synapse formation, Nature 411, 489-494 (2001).

32. E. Hobeika, P. C. Maity, H. Jumaa, Control of B Cell Responsiveness by Isotype and Structural Elements of the Antigen Receptor, Trends in Immunology 37, 310-320 (2016).

33. R. Übelhart, E. Hug, M. P. Bach, T. Wossning, M. Dühren-von Minden, A. H. C. Horn, D. Tsiantoulas, K. Kometani, T. Kurosaki, C. J. Binder, H. Sticht, L. Nitschke, M. Reth, H. Jumaa, Responsiveness of B cells is regulated by the hinge region of IgD, Nature Immunology 16, 534-543 (2015).

34. J. N. Arnold, M. R. Wormald, R. B. Sim, P. M. Rudd, R. A. Dwek, The Impact of Glycosylation on the Biological Function and Structure of Human Immunoglobulins, Annu. Rev. Immunol. 25, 21-50 (2007).

35. E. Maverakis, K. Kim, M. Shimoda, M. E. Gershwin, F. Patel, R. Wilken, S. Raychaudhuri, L. R. Ruhaak,

C. B. Lebrilla, Glycans in the immune system and The Altered Glycan Theory of Autoimmunity: A critical review, Journal of Autoimmunity 57, 1-13 (2015).

36. K.-T. Shade, R. Anthony, Antibody Glycosylation and Inflammation, Antibodies 2, 392-414 (2013).

37. V. Coelho, S. Krysov, A. M. Ghaemmaghami, M. Emara, K. N. Potter, P. Johnson, G. Packham, L. Martinez-Pomares, F. K. Stevenson, in (2010).

38. F. Vuillier, G. Dumas, C. Magnac, M.-C. Prevost, A. I. Lalanne, P. Oppezzo, E. Melanitou, G. Dighiero, B. Payelle-Brogard, Lower levels of surface B-cell-receptor expression in chronic lymphocytic leukemia are associated with glycosylation and folding defects of the mu and CD79a chains, Blood 105, 2933-2940 (2005). 39. T. Kurosaki, Functional dissection of BCR signaling pathways, Current Opinion in Immunology 12, 276 $281(2000)$.

40. L. B. King, B. D. Freedman, B-lymphocyte calcium influx, Immunol. Rev. 231, 265-277 (2009). 
41. Y. Baba, M. Matsumoto, T. Kurosaki, Calcium signaling in B cells: Regulation of cytosolic $\mathrm{Ca}^{2+}$ increase and its sensor molecules, STIM1 and STIM2, Molecular Immunology, 1-5 (2013).

42. V. W. Chan, C. A. Lowell, A. L. DeFranco, Defective negative regulation of antigen receptor signaling in Lyn-deficient B lymphocytes, Curr. Biol. 8, 545-553 (1998).

43. C. Lamagna, Y. Hu, A. L. DeFranco, C. A. Lowell, B Cell-Specific Loss of Lyn Kinase Leads to Autoimmunity, J. Immunol. 192, 919-928 (2014).

44. K. P. Lam, R. Kühn, K. Rajewsky, In vivo ablation of surface immunoglobulin on mature B cells by inducible gene targeting results in rapid cell death, Cell 90, 1073-1083 (1997).

45. E. Levit-Zerdoun, M. Becker, R. Pohlmeyer, I. Wilhelm, P. C. Maity, K. Rajewsky, M. Reth, E. Hobeika, Survival of Ig $\alpha$-Deficient Mature B Cells Requires BAFF-R Function, J. Immunol. 196, 2348-2360 (2016).

46. M. Kraus, L. I. Pao, A. Reichlin, Y. Hu, B. Canono, J. C. Cambier, M. C. Nussenzweig, K. Rajewsky, Interference with Immunoglobulin (Ig) a Immunoreceptor Tyrosine-based Activation Motif (ITAM) Phosphorylation Modulates or Blocks B cell development, Depending on the Availability of an Igb Cytoplasmic Tail, J Exp Med 194, 455-470 (2001).

47. C. Lutz, B. Ledermann, M. H. Kosco-Vilbois, A. F. Ochsenbein, R. M. Zinkernagel, G. Köhler, F. Brombacher, IgD can largely substitute for loss of IgM function in B cells, Nature 393, 797-801 (1998).

48. J. G. Monroe, W. L. Havran, J. C. Cambier, B lymphocyte activation: entry into cell cycle is accompanied by decreased expression of IgD but not IgM, Eur. J. Immunol. 13, 208-213 (1983).

49. K. A. Fairfax, L. M. Corcoran, C. Pridans, N. D. Huntington, A. Kallies, S. L. Nutt, D. M. Tarlinton, Different Kinetics of Blimp-1 Induction in B Cell Subsets Revealed by Reporter Gene, J. Immunol. 178, 41044111 (2007).

50. A. Kallies, J. Hasbold, D. M. Tarlinton, W. Dietrich, L. M. Corcoran, P. D. Hodgkin, S. L. Nutt, Plasma Cell Ontogeny Defined by Quantitative Changes in Blimp-1 Expression, J Exp Med 200, 967-977 (2004).

51. P. C. Maity, A. Blount, H. Jumaa, O. Ronneberger, B. F. Lillemeier, M. Reth, B cell antigen receptors of the IgM and IgD classes are clustered in different protein islands that are altered during B cell activation, Sci Signal 8 (2015), doi:10.1126/scisignal.2005887.

52. K. Kläsener, P. C. Maity, E. Hobeika, J. Yang, M. Reth, B cell activation involves nanoscale receptor reorganizations and inside-out signaling by Syk, eLife 3, 1497-17 (2014).

53. P. Hou, E. Araujo, T. Zhao, M. Zhang, D. Massenburg, M. Veselits, C. Doyle, A. R. Dinner, M. R. Clark, D. Nemazee, Ed. B Cell Antigen Receptor Signaling and Internalization Are Mutually Exclusive Events, PLoS Biol 4, e200-12 (2006).

54. P. K. A. Mongini, S. Tolani, R. J. Fattah, J. K. Inman, Antigen receptor triggered upregulation of CD86 and CD80 in human B cells: augmenting role of the CD21/CD19 co-stimulatory complex and IL-4, Cell. Immunol. 216, 50-64 (2002).

55. D. Frasca, M. Romero, A. Diaz, S. Alter-Wolf, M. Ratliff, A. M. Landin, R. L. Riley, B. B. Blomberg, A Molecular Mechanism for TNF-alpha-Mediated Downregulation of B Cell Responses, J. Immunol. 188, 279 286 (2011).

56. R. C. Rickert, J. Jellusova, A. V. Miletic, Signaling by the tumor necrosis factor receptor superfamily in B-cell biology and disease, Immunol. Rev. 244, 115-133 (2011).

57. C. J. Hanson, M. D. Bootman, H. L. Roderick, Cell Signalling: IP3 Receptors Channel Calcium into Cell Death, Current Biology 14, R933-R935 (2004).

58. A. M. Scharenberg, L. A. Humphries, D. J. Rawlings, Calcium signalling and cell-fate choice in B cells, Nat Rev Immunol 7, 778-789 (2007).

59. D. Donjerković, D. W. Scott, Activation-induced cell death in B lymphocytes, Cell Research 10, 179-192 (2000).

60. I. Ceballos-Olvera, M. Sahoo, M. A. Miller, L. D. Barrio, F. Re, D. J. Philpott, Ed. Inflammasomedependent Pyroptosis and IL-18 Protect against Burkholderia pseudomallei Lung Infection while IL-1 $\beta$ Is Deleterious, PLoS Pathog 7, e1002452-13 (2011).

61. R. Rosales-Reyes, A. Pérez-López, C. Sánchez-Gómez, R. R. Hernández-Mote, D. Castro-Eguiluz, V. Ortiz-Navarrete, C. M. Alpuche-Aranda, Salmonella infects B cells by macropinocytosis and formation of 
spacious phagosomes but does not induce pyroptosis in favor of its survival, Microbial Pathogenesis 52, $367-$ 374 (2012).

62. L. Schoenlaub, R. Cherla, Y. Zhang, G. Zhang, C. R. Roy, Ed. Coxiella burnetii Avirulent Nine Mile Phase II Induces Caspase-1-Dependent Pyroptosis in Murine Peritoneal B1a B Cells, Infection and Immunity 84, 3638-3654 (2016).

63. V. A. K. Rathinam, K. A. Fitzgerald, Inflammasome Complexes: Emerging Mechanisms and Effector Functions, Cell 165, 792-800 (2016).

64. V. Y. Melnikov, S. Faubel, B. Siegmund, M. S. Lucia, D. Ljubanovic, C. L. Edelstein, Neutrophilindependent mechanisms of caspase-1- and IL-18-mediated ischemic acute tubular necrosis in mice, Journal of Clinical Investigation 110, 1083-1091 (2002).

65. T. M. Caserta, A. N. Smith, A. D. Gultice, M. A. Reedy, T. L. Brown, Q-VD-OPh, a broad spectrum caspase inhibitor with potent antiapoptotic properties, Apoptosis 8, 345-352 (2003).

66. B. Boulianne, O. L. Rojas, D. Haddad, A. Zaheen, A. Kapelnikov, T. Nguyen, C. Li, R. Hakem, J. L. Gommerman, A. Martin, AID and Caspase 8 Shape the Germinal Center Response through Apoptosis, $J$. Immunol. 191, 5840-5847 (2013).

67. P. Lee, Z. Zhu, J. Hachmann, T. Nojima, D. Kitamura, G. Salvesen, R. C. Rickert, Differing Requirements for MALT1 Function in Peripheral B Cell Survival and Differentiation, J. Immunol. 198, 1066-1080 (2016).

68. W. P. Lafuse, R. Gearinger, S. Fisher, C. Nealer, A. R. Mackos, M. T. Bailey, Exposure to a Social Stressor Induces Translocation of Commensal Lactobacilli to the Spleen and Priming of the Innate Immune System, $J$. Immunol. 198, 2383-2393 (2017).

69. S. Rose, A. Misharin, H. Perlman, A novel Ly6C/Ly6G-based strategy to analyze the mouse splenic myeloid compartment, Cytometry 81A, 343-350 (2011).

70. M. Kraus, M. B. Alimzhanov, N. Rajewsky, K. Rajewsky, Survival of Resting Mature B Lymphocytes Depends on BCR Signaling via the Ig $\alpha / \beta$ Heterodimer, Cell 117, 787-800 (2004).

71. G. K. Hirst, The agglutination of red cells by allantoic fluid of chick embryos infected with influenza virus, Science 94, 22-23 (1941).

72. A. Gottschalk, S. M. Patridge, Interaction between simple sugars and amino-acids, Nature 165, 684-685 (1950).

73. T. Schubert, W. Römer, How synthetic membrane systems contribute to the understanding of lipid-driven endocytosis, BBA - Molecular Cell Research, 1-14 (2015).

74. Y.-M. Kim, J. Y.-J. Pan, G. A. Korbel, V. Peperzak, M. Boes, H. L. Ploegh, Monovalent ligation of the B cell receptor induces receptor activation but fails to promote antigen presentation, PNAS 103, 3327-3332 (2006).

75. S. Mukherjee, J. Zhu, J. Zikherman, R. Parameswaran, T. A. Kadlecek, Q. Wang, B. Au-Yeung, H. Ploegh, J. Kuriyan, J. Das, A. Weiss, Monovalent and multivalent ligation of the B cell receptor exhibit differential dependence upon Syk and Src family kinases, Sci Signal 6, ra1-ral (2013).

76. M. Becker, E. Hobeika, H. Jumaa, M. Reth, P. C. Maity, CXCR4 signaling and function require the expression of the IgD-class B-cell antigen receptor, Proc. Natl. Acad. Sci. U.S.A. 114, 5231-5236 (2017).

77. J. Z. Chen, A. M. Stall, L. A. Herzenberg, L. A. Herzenberg, L. A. Herzenberg, Differences in glycoprotein complexes associated with IgM and IgD on normal murine B cells potentially enable transduction of different signals, EMBO J. 9, 2117-2124 (1990).

78. G. J. Silverman, C. S. Goodyear, A Model B-Cell Superantigen and the Immunobiology of B Lymphocytes, Clinical Immunology 102, 117-134 (2002).

79. G. J. Silverman, C. S. Goodyear, Confounding B-cell defences: lessons from a staphylococcal superantigen, Nat Rev Immunol 6, 465-475 (2006).

80. J. Jellusova, A. V. Miletic, M. H. Cato, W.-W. Lin, Y. Hu, G. A. Bishop, M. J. Shlomchik, R. C. Rickert, Context-Specific BAFF-R Signaling by the NF-kappaB and PI3K Pathways, CellReports 5, 1022-1035 (2013).

81. E. Hobeika, E. Levit-Zerdoun, V. Anastasopoulou, R. Pohlmeyer, S. Altmeier, A. Alsadeq, M.-W. Dobenecker, R. Pelanda, M. Reth, CD19 and BAFF-R can signal to promote B-cell survival in the absence of Syk, EMBO J. 34, 925-939 (2015). 
82. X. Chen-Deutsch, A. Kutner, J. S. Harrison, G. P. Studzinski, The pan-caspase inhibitor Q-VD-OPh has anti-leukemia effects and can interact with vitamin D analogs to increase HPK1 signaling in AML cells, Leukemia Research 36, 884-888 (2012).

83. T. G. Nguyen, J. M. Morris, Signals from activation of B-cell receptor with anti-IgD can override the stimulatory effects of excess BAFF on mature B cells in vivo, Immunology Letters 161, 157-164 (2014).

84. S. E. Spurgeon, G. Coffey, L. B. Fletcher, R. Burke, J. W. Tyner, B. J. Druker, A. Betz, F. DeGuzman, Y. Pak, D. Baker, A. Pandey, S. J. Hollenbach, U. Sinha, M. M. Loriaux, The selective SYK inhibitor P505-15 (PRT062607) inhibits B cell signaling and function in vitro and in vivo and augments the activity of fludarabine in chronic lymphocytic leukemia, Journal of Pharmacology and Experimental Therapeutics 344, 378-387 (2013).

85. P. M. Higgins, Splenomegaly in acute infections due to group A streptococci and viruses, Epidemiology and Infection 109, 199-209 (1992).

86. P. A. Nikitin, A. M. Price, K. McFadden, C. M. Yan, M. A. Luftig, L. Zhang, Ed. Mitogen-Induced B-Cell Proliferation Activates Chk2-Dependent G1/S Cell Cycle Arrest, PLoS ONE 9, e87299-8 (2014).

87. J. S. Turner, M. Marthi, Z. L. Benet, I. Grigorova, Transiently antigen-primed B cells return to naive-like state in absence of T-cell help, Nature Communications 8, 1-11 (2017).

88. B. Nardelli, O. Belvedere, V. Roschke, P. A. Moore, H. S. Olsen, T. S. Migone, S. Sosnovtseva, J. A. Carrell, P. Feng, J. G. Giri, D. M. Hilbert, Synthesis and release of B-lymphocyte stimulator from myeloid cells, Blood 97, 198-204 (2001).

89. P. Schneider, F. Mackay, V. Steiner, K. Hofmann, J.-L. Bodmer, N. Holler, C. Ambrose, P. Lawton, S. Bixler, H. Acha-Orbea, D. Valmori, P. Romero, C. Werner-Favre, R. H. Zubler, J. L. Browning, J. Tschopp, BAFF, a Novel Ligand of the Tumor Necrosis Factor Family, Stimulates B Cell Growth, J Exp Med 189, 1747 1756 (1999).

90. C. Chaparro, J. Maurer, C. Gutierrez, M. Krajden, C. Chan, T. Winton, S. Keshavjee, M. Scavuzzo, E. Tullis, M. Hutcheon, S. Kesten, Infection with Burkholderia cepacia in cystic fibrosis: outcome following lung transplantation, Am. J. Respir. Crit. Care Med. 163, 43-48 (2001).

91. H. Funken, K.-M. Bartels, S. Wilhelm, M. Brocker, M. Bott, M. Bains, R. E. W. Hancock, F. Rosenau, K.E. Jaeger, P. Cornelis, Ed. Specific Association of Lectin LecB with the Surface of Pseudomonas aeruginosa: Role of Outer Membrane Protein OprF, PLoS ONE 7, e46857-8 (2012).

92. P. L. Wagner, J. Livny, M. N. Neely, D. W. K. Acheson, D. I. Friedman, M. K. Waldor, Bacteriophage control of Shiga toxin 1 production and release by Escherichia coli, Molecular Microbiology 44, 957-970 (2002).

93. P. L. Wagner, D. W. Acheson, M. K. Waldor, Human neutrophils and their products induce Shiga toxin production by enterohemorrhagic Escherichia coli, Infection and Immunity 69, 1934-1937 (2001).

94. S. B. Calderwood, J. J. Mekalanos, Genetic Analysis of Regulation and Structure of Shiga-Like Toxin I in Escherichia Coli (Springer US, Boston, MA, 1990), pp. 57-64.

95. C. Chemani, A. Imberty, S. de Bentzmann, M. Pierre, M. Wimmerova, B. P. Guery, K. Faure, Role of LecA and LecB Lectins in Pseudomonas aeruginosa-Induced Lung Injury and Effect of Carbohydrate Ligands, Infection and Immunity 77, 2065-2075 (2009).

96. K. Nothelfer, P. J. Sansonetti, A. Phalipon, Pathogen manipulation of B cells: the best defence is a good offence, Nature Reviews Microbiology 13, 173-184 (2015).

97. K. Nothelfer, E. T. Arena, L. Pinaud, M. Neunlist, B. Mozeleski, I. Belotserkovsky, C. Parsot, P. Dinadayala, A. Burger-Kentischer, R. Raqib, P. J. Sansonetti, A. Phalipon, B lymphocytes undergo TLR2dependent apoptosis upon Shigella infection, J Exp Med 211, 1215-1229 (2014).

98. K. Chen, W. Xu, M. Wilson, B. He, N. W. Miller, E. Bengtén, E.-S. Edholm, P. A. Santini, P. Rath, A. Chiu, M. Cattalini, J. Litzman, J. B Bussel, B. Huang, A. Meini, K. Riesbeck, C. Cunningham-Rundles, A. Plebani, A. Cerutti, Immunoglobulin D enhances immune surveillance by activating antimicrobial, proinflammatory and B cell-stimulating programs in basophils, Nature Immunology 10, 889-898 (2009).

99. A. J. Ulloa-Morales, C. S. Goodyear, G. J. Silverman, Essential Domain-Dependent Roles Within Soluble IgG for in vivo Superantigen Properties of Staphylococcal Protein A: Resolving the B-Cell Superantigen Paradox, Front. Immunol. 9, 1840-12 (2018). 
100. W. Li, Q. Liu, Y. Pang, J. Jin, H. Wang, H. Cao, Z. Li, X. Wang, B. Ma, Y. Chi, R. Wang, A. Kondo, J. $\mathrm{Gu}, \mathrm{N}$. Taniguchi, Core Fucosylation of uHeavy Chains Regulates Assembly and Intracellular Signaling of Precursor B Cell Receptors, Journal of Biological Chemistry 287, 2500-2508 (2012).

101. A. L. Morrow, J. Meinzen-Derr, P. Huang, K. R. Schibler, T. Cahill, M. Keddache, S. G. Kallapur, D. S. Newburg, M. Tabangin, B. B. Warner, X. Jiang, Fucosyltransferase 2 Non-Secretor and Low Secretor Status Predicts Severe Outcomes in Premature Infants, The Journal of Pediatrics 158, 745-751 (2011).

102. B. Carlsson, E. Kindberg, J. Buesa, G. E. Rydell, M. F. Lidón, R. Montava, R. A. Mallouh, A. Grahn, J. Rodríguez-Díaz, J. Bellido, A. Arnedo, G. Larson, L. Svensson, B. A. Lopman, Ed. The G428A Nonsense Mutation in FUT2 Provides Strong but Not Absolute Protection against Symptomatic GII.4 Norovirus Infection, PLOS ONE 4, e5593-10 (2009).

103. M. Fumagalli, R. Cagliani, U. Pozzoli, S. Riva, G. P. Comi, G. Menozzi, N. Bresolin, M. Sironi, Widespread balancing selection and pathogen-driven selection at blood group antigen genes, Genome Research 19, 199-212 (2008).

104. R. F. Villar, J. Patel, G. C. Weaver, M. Kanekiyo, A. K. Wheatley, H. M. Yassine, C. E. Costello, K. B. Chandler, P. M. McTamney, G. J. Nabel, A. B. McDermott, J. R. Mascola, S. A. Carr, D. Lingwood, Reconstituted B cell receptor signaling reveals carbohydrate- dependent mode of activation, Sci. Rep., 1-11 (2016).

105. R. Pelanda, U. Braun, E. Hobeika, M. C. Nussenzweig, M. Reth, B cell progenitors are arrested in maturation but have intact VDJ recombination in the absence of Ig-alpha and Ig-beta, J. Immunol. 169, 865872 (2002).

106. L. Nitschke, M. H. Kosco, G. Köhler, M. C. Lamers, Immunoglobulin D-deficient mice can mount normal immune responses to thymus-independent and -dependent antigens, PNAS 90, 1887-1891 (1993).

107. M. Inaoki, S. Sato, B. C. Weintraub, C. C. Goodnow, T. F. Tedder, CD19-regulated signaling thresholds control peripheral tolerance and autoantibody production in B lymphocytes, J Exp Med 186, 1923-1931 (1997).

108. M. L. Hibbs, D. M. Tarlinton, J. Armes, D. Grail, G. Hodgson, Multiple defects in the immune system of Lyn-deficient mice, culminating in autoimmune disease, Cell 83, 301-311 (1995).

109. S. Sugawara, M. Hosono, Y. Ogawa, M. Takayanagi, K. Nitta, Catfish egg lectin causes rapid activation of multidrug resistance 1 P-glycoprotein as a lipid translocase, Biol. Pharm. Bull. 28, 434-441 (2005).

Acknowledgments: We thank M. Becker, P. C. Maity, and the whole Reth laboratory for material and technical support, particularly for access to the animal facility of the Max Planck Institute of Immunology and Epigenetics, Freiburg, Germany. Furthermore, we appreciate support by the BIOSS Toolbox, especially P. Salavei, in the purification of the bacterial lectins. We further thank P. Hennecke for helpful discussions during thesis committee meetings, as well as S. Samodelov and R. Pohlmeyer for proofreading the manuscript. We would like to acknowledge A. Nyström of the Bruckner-Tudermann group and C. Molnár from the Erlacher group at the University Medical Centre Freiburg for their supply of the murine WT spleens for some in vitro experiments. We also thank J. M. Weiss from the Erlacher group for her help with the myeloid gating strategy and G. Andrieux for the evaluation of the statistical analysis. Funding: The research group of W.R. was supported by the Excellence Initiative of the German Research Foundation (EXC 294 and GSC-4), the Ministry of Science, Research and the Arts Baden-Württemberg (Az: 33-7532.20), and by a starting grant from the European Research Council (Programme "Ideas", ERC-2011-StG 282105-lec\&lip2invade). A.I. and W.R. acknowledge support by the French National Research Agency (ANR) and the German Federal Ministry of Education and Research (BMBF) in the framework of the EU ERASynBio project SynGlycTis (ANR-14SYNB-0002-02; BMBF 031A464). A.I. was further supported by Glyco@Alps (ANR-15-IDEX-02). This study was partially funded by the German Research Agency (DFG) by the grants RTG 2202 and RO 4341/21. I.W. and R.U. were funded by the Excellence Initiative of the DFG (GSC-4, Spemann Graduate School) and E.H. was partly supported by the SFB 1074 P N09. E.L.Z. and M.F. were funded by the International Max Planck Research School for Molecular and Cellular Biology, Max Planck Institute of Immunobiology and Epigenetics, Freiburg, Germany. Author contributions: I.W., W.R. and E.H. designed this interdisciplinary study. The experiments were conducted by I.W., M.F., and J.J. under the supervision of E.H., E.L-Z., and W.R. 
Furthermore, P.M., S.V., and A.L. provided preliminary data. A.I., R.T., and J.C. established the lectin experiments and supervised the respective data analysis. R.U. helped in performing $\mathrm{Ca}^{2+}$ experiments and establishing of methodology. I.W. and E.L-Z. performed data analysis and wrote the manuscript. M.R., H.E., and H.J. contributed with experimental advice and B cell-specific expertise. W.R. and E.H. supervised the study. Competing interests: The authors declare that they have no competing interests. Data and materials availability: All data needed to evaluate the conclusions in the paper are present in the paper or the Supplementary Materials. 
A

SP CD93-

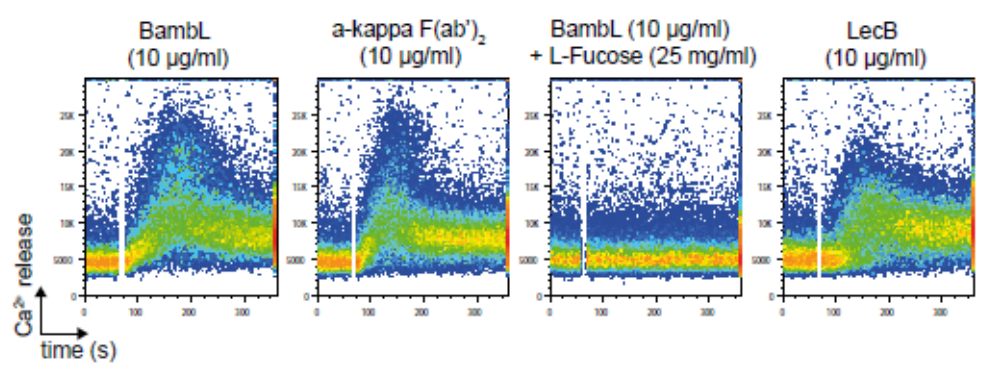

B

$\mathrm{SP}_{\mathrm{CD}} 3^{-}$

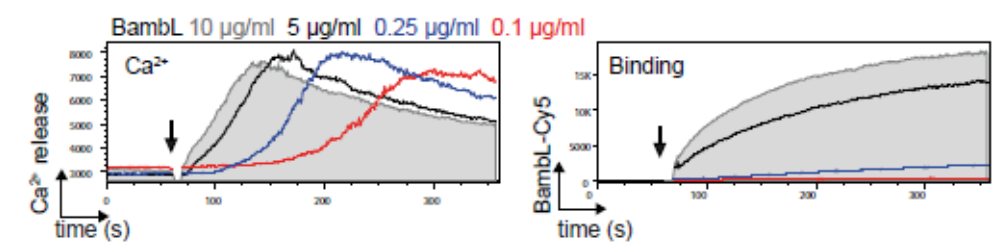

C
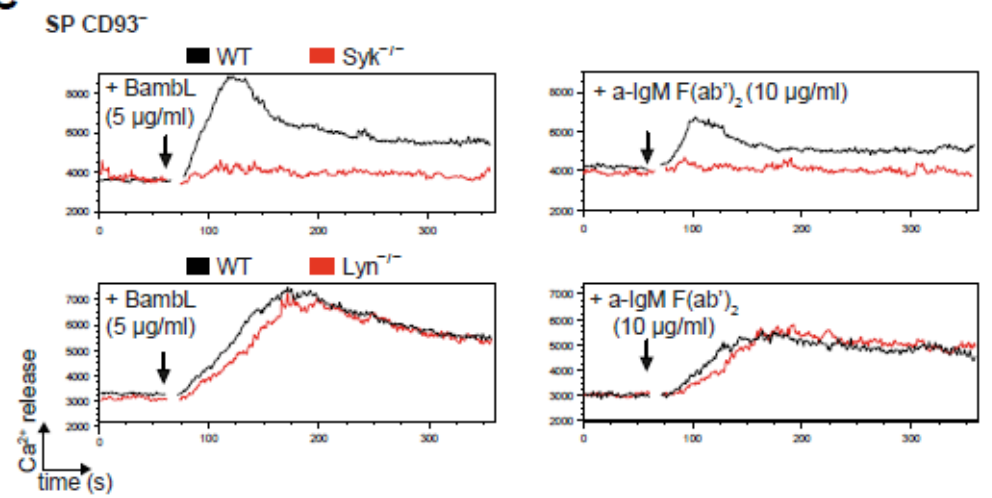

D
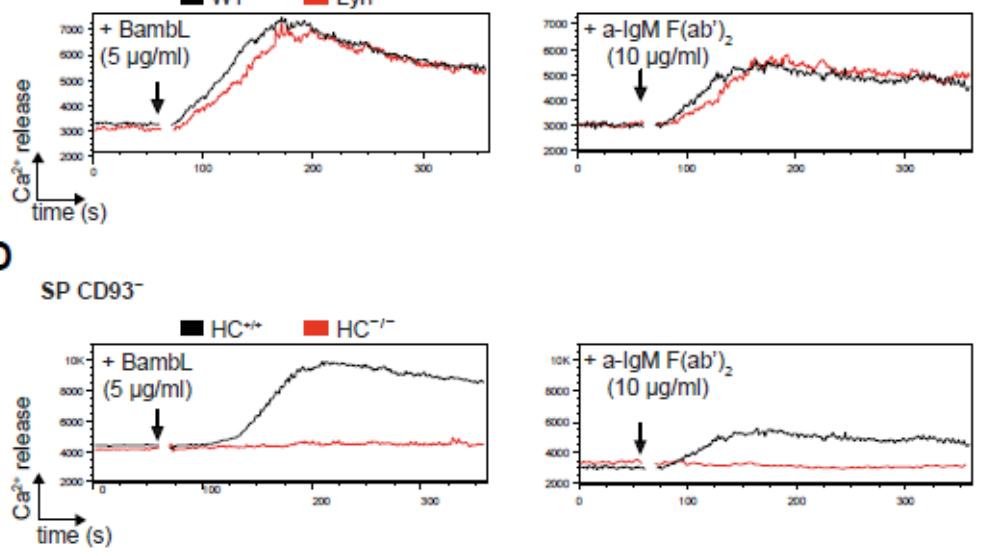

Fig. 1. BCR and Syk are essential for BambL-induced $\mathrm{Ca}^{2+}$ release in primary $\mathrm{B}$ cells. (A) Flow cytometric analysis of $\mathrm{Ca}^{2+}$ release in splenic mature follicular (MFo) B cells from wild type (WT) mice upon stimulation with $10 \mu \mathrm{g} / \mathrm{ml} \mathrm{BambL}, 10 \mu \mathrm{g} / \mathrm{ml} \mathrm{LecB}$, or $10 \mu \mathrm{g} / \mathrm{ml}$ anti-kappa F(ab')2 antibody Ab as a control. BambL preincubated with L-Fucose served as a control for BambL fucose-binding specificity. Data are representative of four independent experiments. (B) Dose-dependent stimulation of MFo B cells with BambL was monitored by flow cytometric analysis of $\mathrm{Ca}^{2+}$ release (left) and binding of BambL (right) to these cells. For the binding analysis, the relative fluorescence of BambL-Cy5 over time is shown. Data are representative of three independent experiments. (C) Flow cytometric analysis of $\mathrm{Ca}^{2+}$ release in WT (black) and $\mathrm{Syk}^{-/-}$(red; top) or $\mathrm{Lyn}^{-/-}$(red; bottom) MFo B cells, stimulated with $10 \mu \mathrm{g} / \mathrm{ml}$ BambL (left) or $10 \mu \mathrm{g} / \mathrm{ml}$ anti-IgM F(ab') $2 \mathrm{Ab}$ (right). Data are representative of three independent experiments. (D) Flow cytometric analysis of $\mathrm{Ca}^{2+}$ release in BCR-negative B cells ( $\mathrm{HC}^{-/-}$, red) as well as in BCR-sufficient B cells from the same mouse ( $\mathrm{HC}^{+/+}$, black). Cells were stimulated with $10 \mu \mathrm{g} / \mathrm{ml} \mathrm{BambL}$ (left) or $10 \mu \mathrm{g} / \mathrm{ml}$ anti-IgM F(ab')2 (right). Data are representative of four independent experiments. The gating strategy for $\mathrm{HC}^{-/}$cells and the $\mathrm{HC}^{+/+}$control is depicted in fig. S1C. 
A
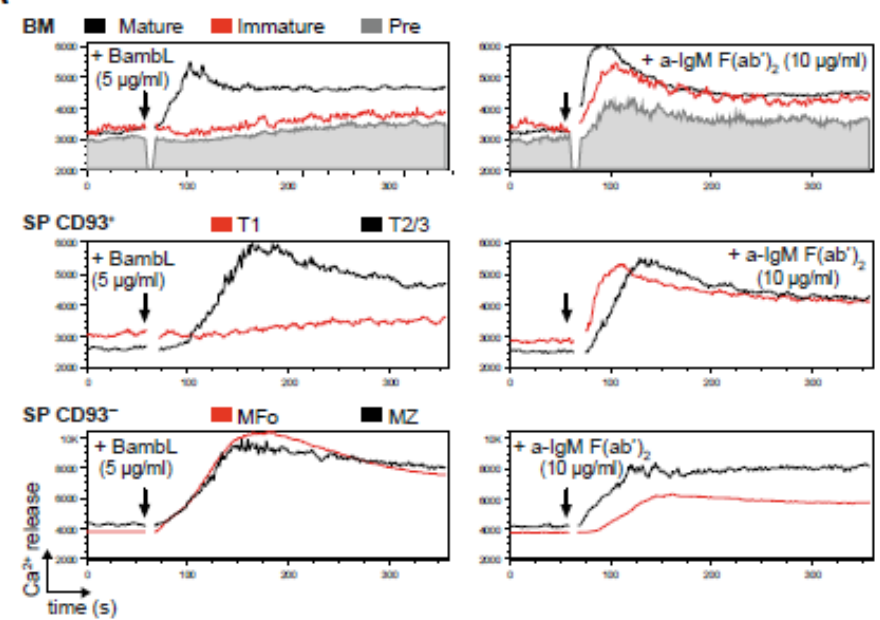

B
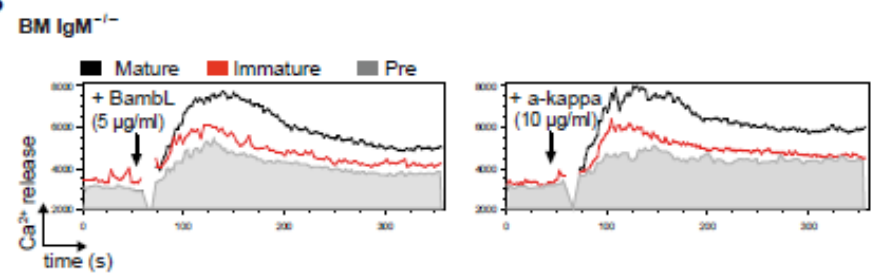

C

LPS-derived plasmablasts

Day4
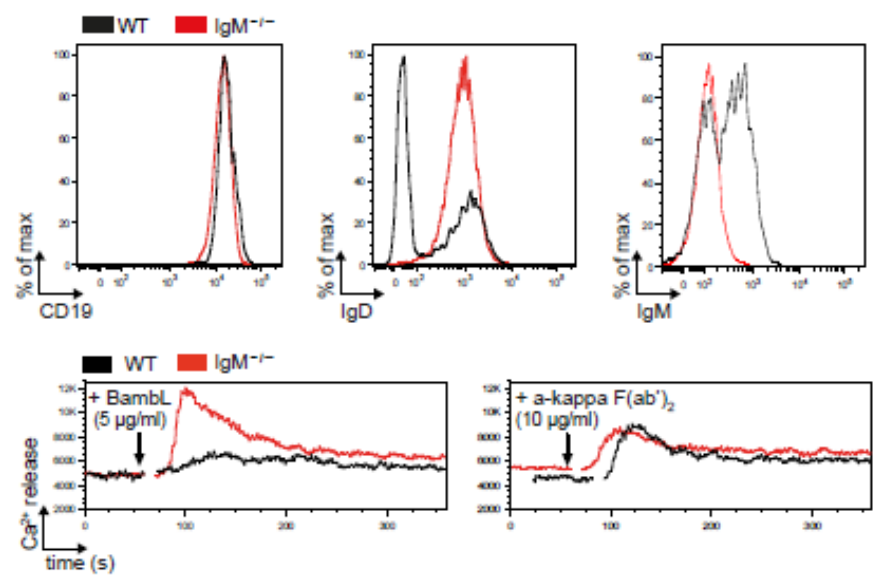

Fig. 2. IgD-expressing $B$ cell subsets release $\mathrm{Ca}^{2+}$ upon treatment with BambL. (A) Flow cytometric analysis of $\mathrm{Ca}^{2+}$ release in WT B cells at different developmental stages: BM-derived pre-B cells (gray), immature B cells (red), and mature B cells (black). Cells were stimulated with BambL $(5 \mu \mathrm{g} / \mathrm{ml}$, left) or anti$\operatorname{IgM~F}\left(\mathrm{ab}^{\prime}\right)_{2}(10 \mu \mathrm{g} / \mathrm{ml}$, right). The BM gating strategy is shown in fig. S2A. The splenic (SP) subsets are displayed in the middle and bottom panels. Middle: $\mathrm{CD}^{+} 3^{+}$; transitional 1 (T1; red), and T2/3 (black line). Bottom: CD93-; mature follicular (MFo; red) B cells; marginal zone (MZ; black) B cells. The SP gating strategy is depicted in fig. S2C. The relative cell surface IgD expression of the respective B cell subtypes is shown in fig. S2D. Data are representative of five independent experiments. (B) Flow cytometric analysis of $\mathrm{Ca}^{2+}$ release in $\mathrm{IgM}^{-/-} \mathrm{BM}$-derived $\mathrm{B}$ cells at different developmental stages. Cells were stimulated with $5 \mu \mathrm{g} / \mathrm{ml} \mathrm{BambL}$ (left) or $10 \mu \mathrm{g} / \mathrm{ml}$ anti-kappa $\mathrm{F}\left(\mathrm{ab}^{\prime}\right) 2$ (right). BM subsets are displayed as pre-B cells (gray), immature B cells (red), and mature B cells (black). Data are representative of three independent experiments. (C) Flow cytometric analysis of $\mathrm{Ca}^{2+}$ release in WT (black) and $\mathrm{IgM}^{-/-}$(red) plasmablasts after 4 days of incubation with LPS. Top: Representative analysis of the cell surface expression of CD19, IgD, and IgM. Cells were selected on the basis of Blimp-GFP and Syndecan-1 (CD138) expression. The cells were stimulated with $5 \mu \mathrm{g} / \mathrm{ml} \mathrm{BambL}$ (bottom left) or $10 \mu \mathrm{g} / \mathrm{ml}$ anti-kappa $\mathrm{F}\left(\mathrm{ab}^{\prime}\right)_{2}$ (bottom right). Data are representative of three independent experiments. 
A
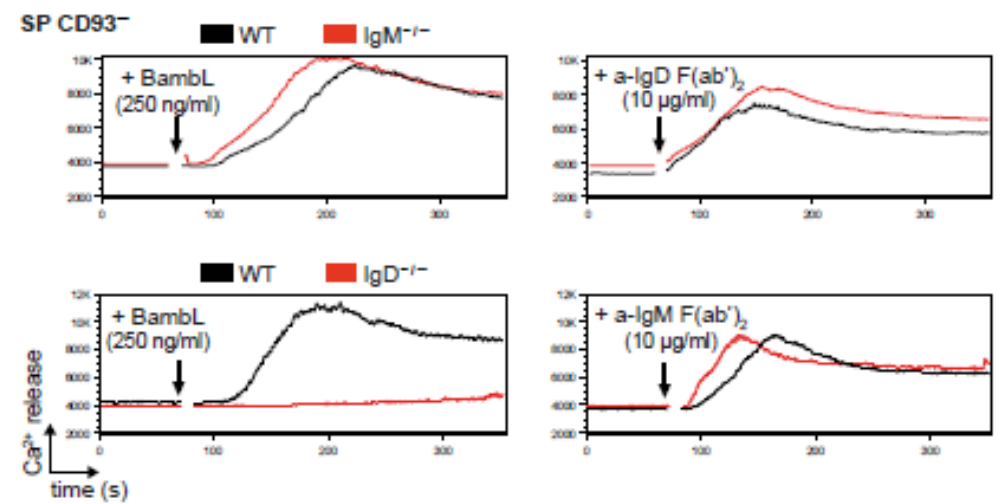

B

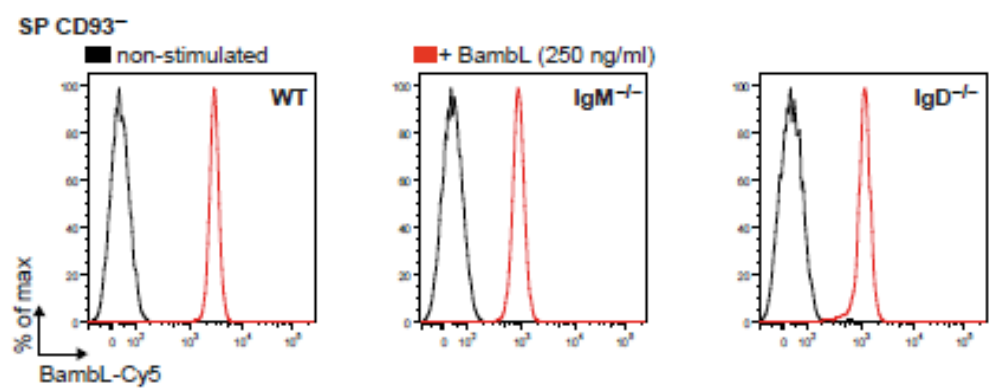

C
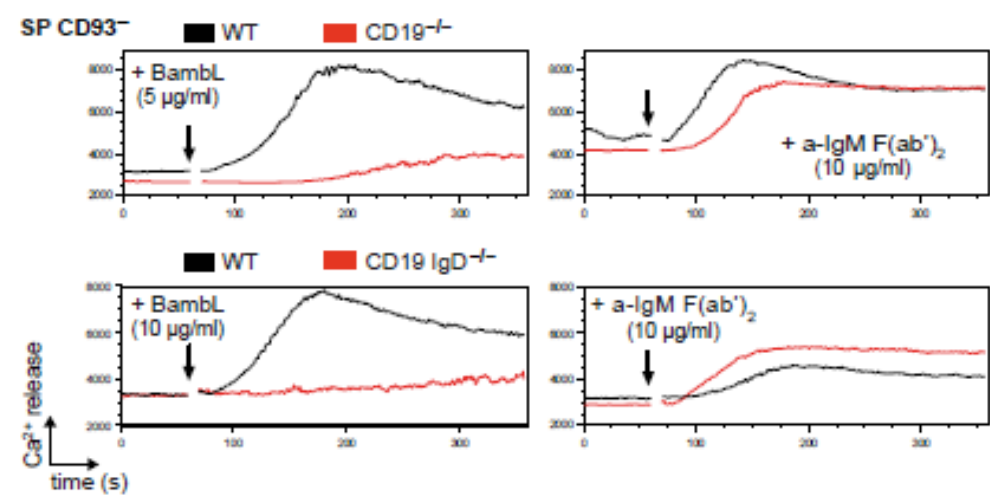

D

SP CD93-
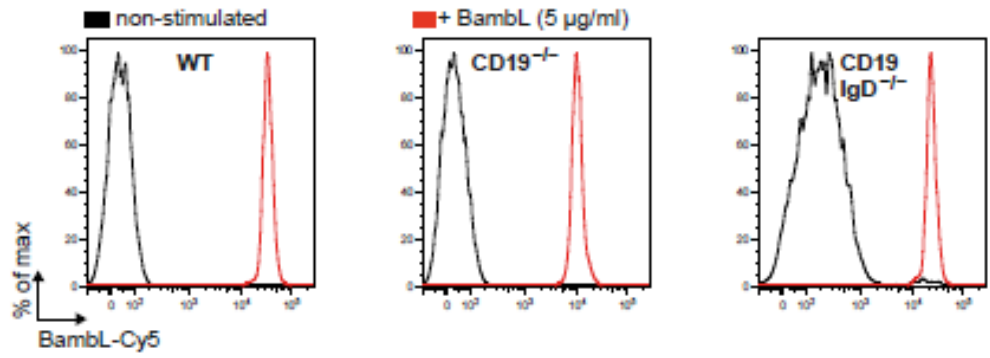

Fig. 3. IgD and CD19 play a major role in BambL-induced signaling. (A) Flow cytometric analysis of $\mathrm{Ca}^{2+}$ release in SP-derived MFo B cells (SP CD93 ${ }^{-}$) from WT (black) and $\operatorname{IgM}^{-/-}$(red; top, $\mathrm{n}=3$ ) and $\operatorname{IgD}^{-/-}$(red; bottom, $\mathrm{n}=4$ ) mice. Cells were stimulated with $250 \mathrm{ng} / \mathrm{ml}$ BambL (left), $10 \mu \mathrm{g} / \mathrm{ml}$ anti-IgD F(ab')2 (top right), or $10 \mu \mathrm{g} / \mathrm{ml}$ anti-IgM F(ab')2 (bottom right). (B) Flow cytometric analysis of BambL-Cy5 binding to the corresponding B cells: WT (left), $\mathrm{IgM}^{-/-}$(middle), and $\mathrm{IgD}^{-/-}$(right). Data are representative of $\mathrm{xxx}$ independent experiments. (C) Flow cytometric analysis of $\mathrm{Ca}^{2+}$ release in WT (black), CD19 ${ }^{-/-}$(red; top, $\mathrm{n}=$ 3 ), and $\mathrm{CD} 19 \mathrm{IgD}^{-1-}$ (red; bottom, $\mathrm{n}=3$ ) B cells. Cells were stimulated with 5 or $10 \mu \mathrm{g} / \mathrm{ml}$ BambL (left) or $10 \mu \mathrm{g} / \mathrm{ml}$ anti-IgM F(ab')2 (right). (D) Flow cytometric analysis of BambL-Cy5 binding to the corresponding $\mathrm{B}$ cells: WT (left), $\mathrm{CD} 19^{-/-}$(middle), and $\mathrm{CD} 19^{+} \mathrm{IgD}^{-/-}$(right). Data are representative of $\mathrm{xxx}$ independent experiments. 
A

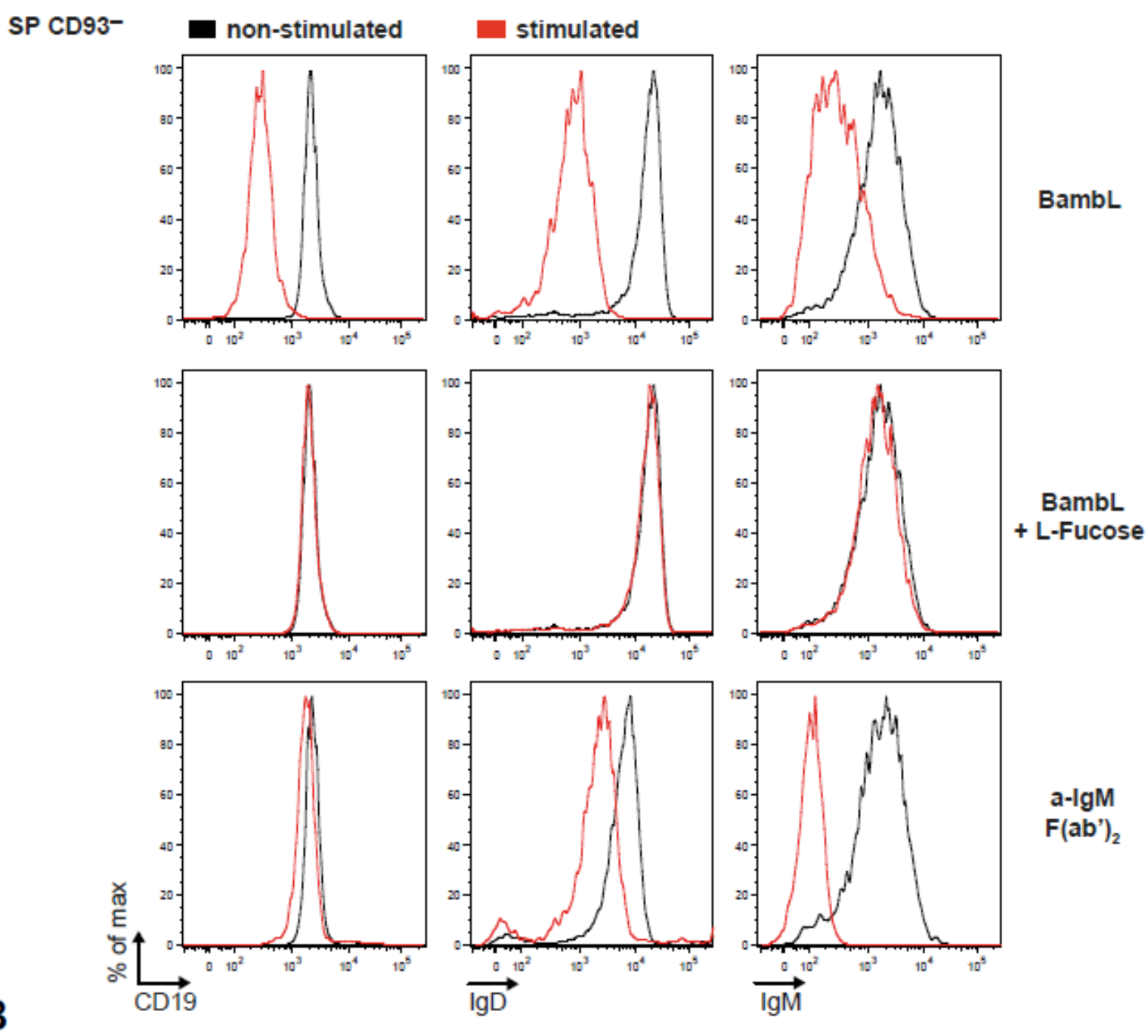

SP CD93-

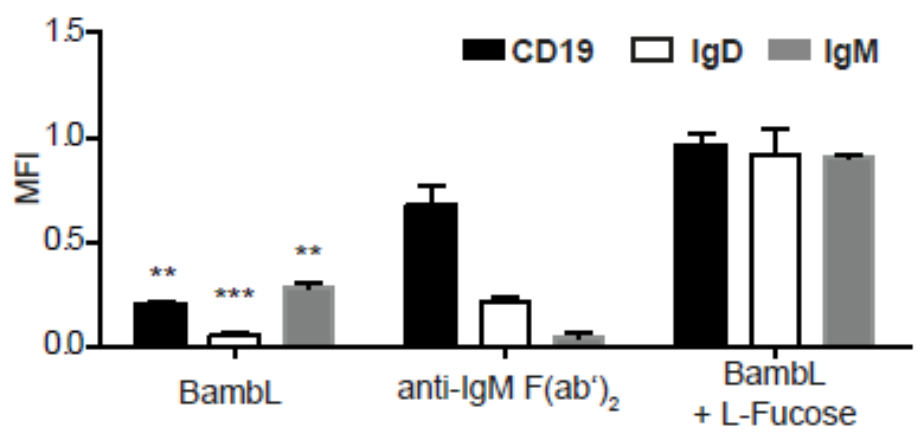

Fig. 4. Binding of BambL leads to the internalization of CD19, IgD, and IgM and can be blocked by soluble L-Fucose. (A) Purified splenic MFo B cells from WT mice were stimulated ex vivo in the presence of $2.5 \mu \mathrm{g} / \mathrm{ml} \mathrm{BambL}, 2.5 \mu \mathrm{g} / \mathrm{ml}$ BambL blocked with $25 \mathrm{mg} / \mathrm{ml} \mathrm{L-Fucose,} \mathrm{or} \mathrm{anti-IgM} \mathrm{F(ab')2} \mathrm{and} \mathrm{analyzed} \mathrm{by}$ flow cytometry after $16 \mathrm{~h}$ of incubation for the cell surface expression of CD19, IgD, IgM, and B220. Unstimulated cells served as a negative control for receptor expression. The combination of BambL and LFucose served as a control for the specificity of BambL. Anti-IgM F(ab') 2 served as a positive control for BCR activation. Viable cells were selected using a fixable viability dye. Data are representative of six independent experiments. (B) Statistical analysis of data shown in (A). Mean values of one representative analysis with at least three technical replicates +/- SD are shown. For statistical analysis, a Student's $t$-test was performed. 
A

$\mathrm{SP} \mathrm{CD}_{3} 3^{-}$

non-stimulated
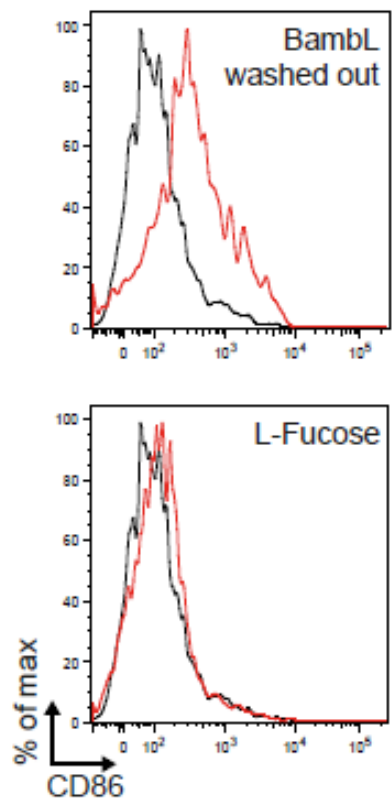

C

$\mathrm{SP} \operatorname{Igd} \mathrm{I}^{-1-}$

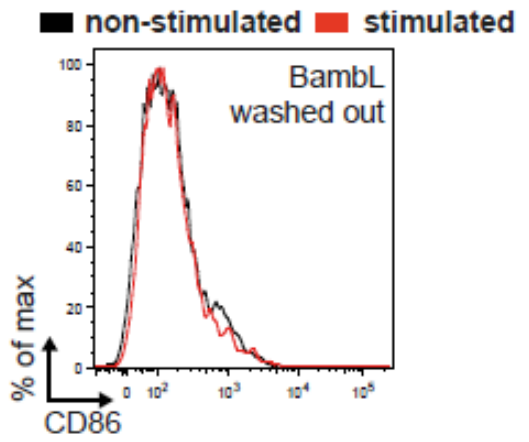

B

SP CD93-
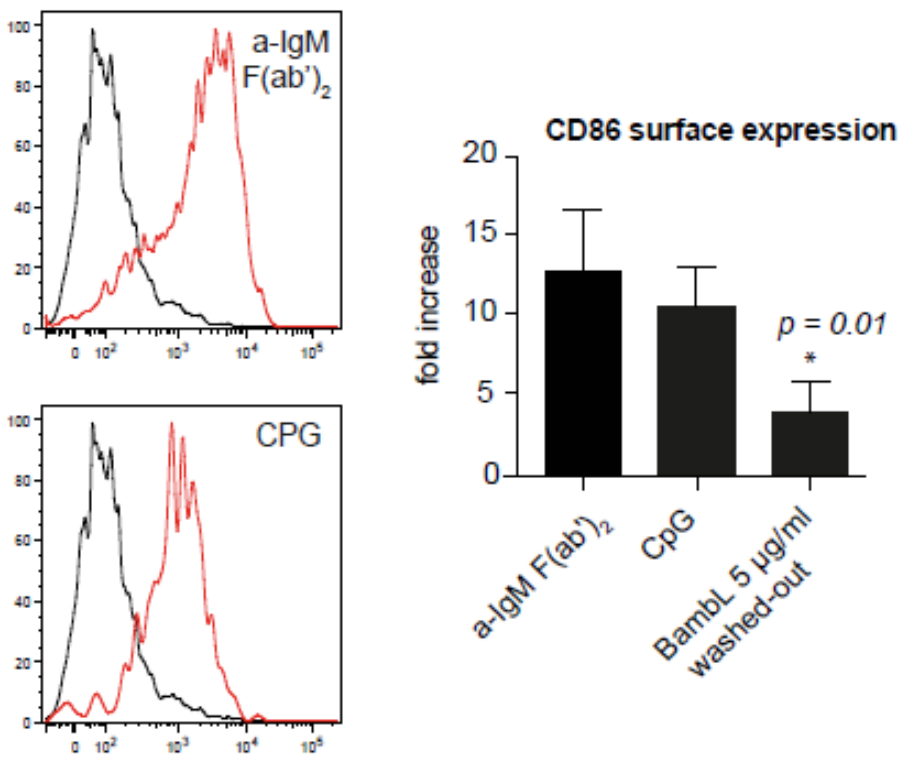
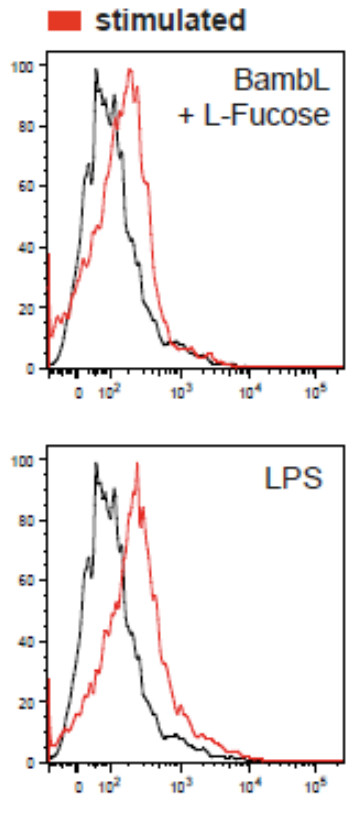

$\mathrm{SPCD} 93^{-}$

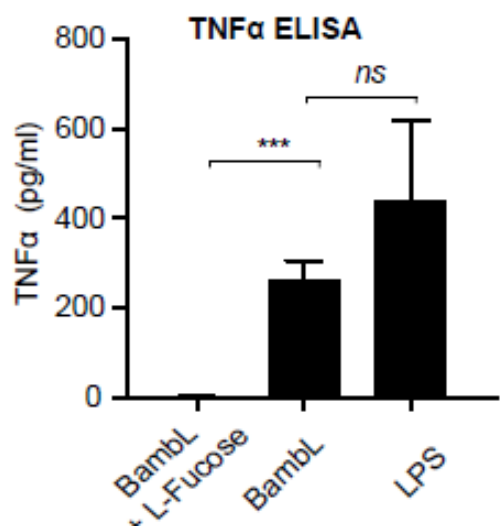

Fig. 5. BambL mediates an increase in CD86 expression and TNF- $\alpha$ secretion. (A) Purified splenic MFo B cells from WT mice were stimulated ex vivo in the presence of the indicated stimuli (see Materials and Methods for concentrations used). CD86 served as an activation marker and its cell surface expression was assessed 16 hours after stimulation. BambL $(5 \mu \mathrm{g} / \mathrm{ml})$ was washed out after $15 \mathrm{~min}$ of stimulation at $37^{\circ} \mathrm{C}$. Unstimulated cells served as a negative control for CD86 expression. The combination of BambL and L-Fucose served as a control for the specificity of BambL. Anti-IgM F(ab')2, LPS, and CpG served as positive controls for BCR and TLR activation. Viable cells were selected using a fixable viability dye. Data are from one experiment and are representative of three independent experiments. (B) Fold-changes in the MFI of CD86 from three independent biological replicate experiments. Cells treated with BambL were compared to the untreated control cells. (C) BCR-negative, Ig $\alpha^{-/}$B cells were treated as described in (A) and examined by flow cytometry to determine the cell surface expression of CD86. The cells were obtained 10 days after tamoxifen treatment. Data are representative of xxx experiments. (D) B cells were stimulated for 16 hours with BambL $(2.5 \mu \mathrm{g} / \mathrm{ml})$, LPS $(10 \mu \mathrm{g} / \mathrm{ml})$, or BambL $(2.5 \mu \mathrm{g} / \mathrm{ml})+$ L-Fucose $(25 \mathrm{mg} / \mathrm{ml})$. The cell culture media were collected and analyzed by ELISA to determine the amount of TNF- $\alpha$ released. Data are means \pm SD of three independent experiments and were analyzed by Student's $t$-test was used to assess the statistical significance of observed differences. 


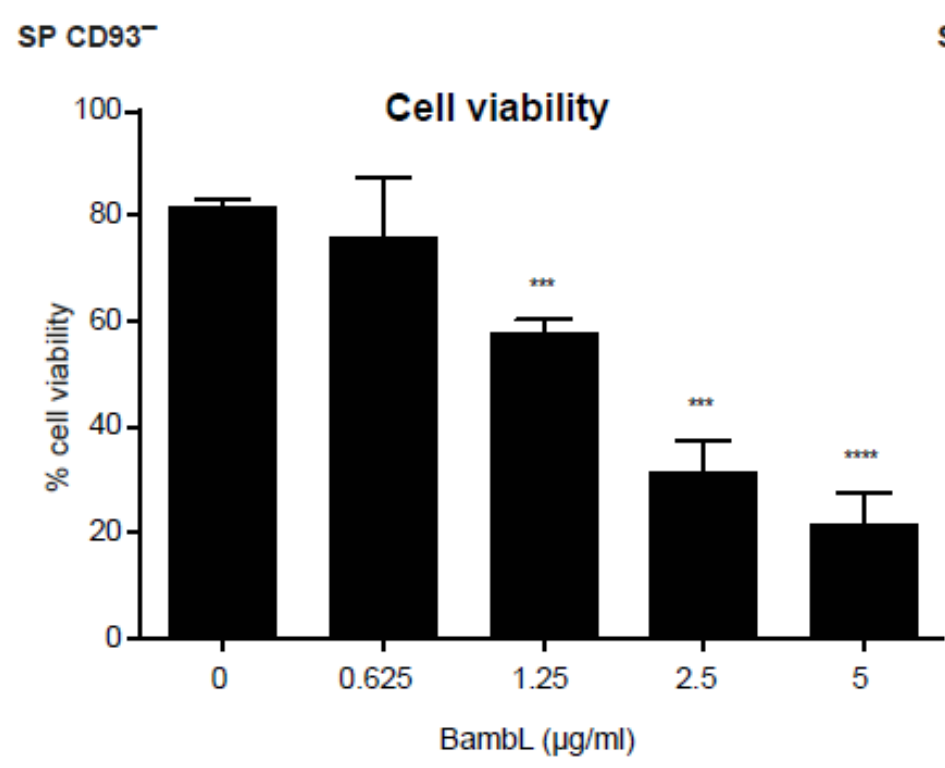

C $\mathrm{SP} \mathrm{CD}^{-} 3^{-}$

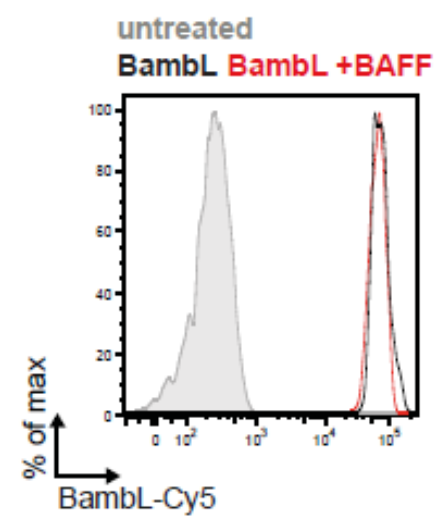

\section{$\mathrm{SP} \mathrm{CD}^{-} 3^{-}$}
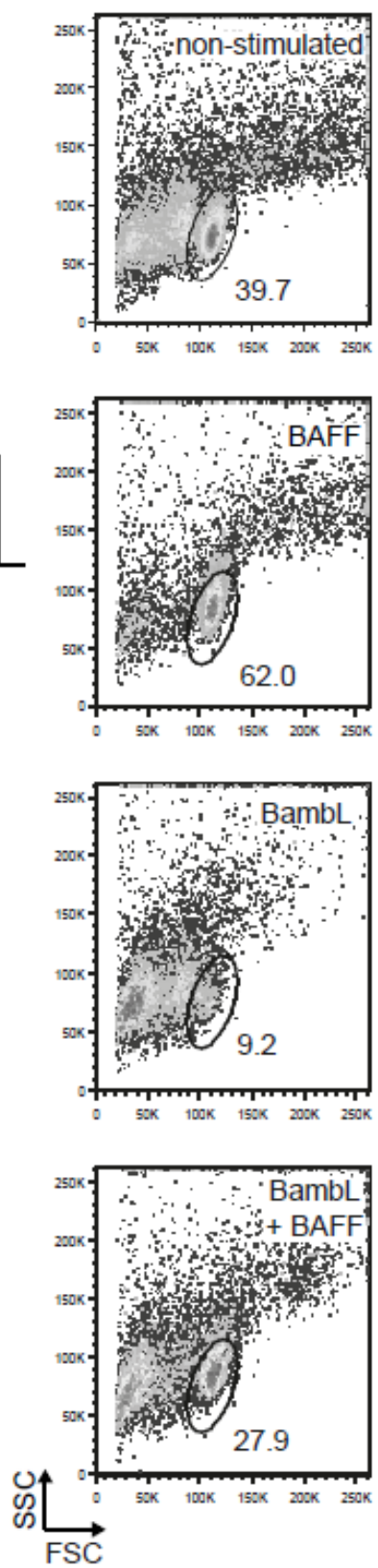
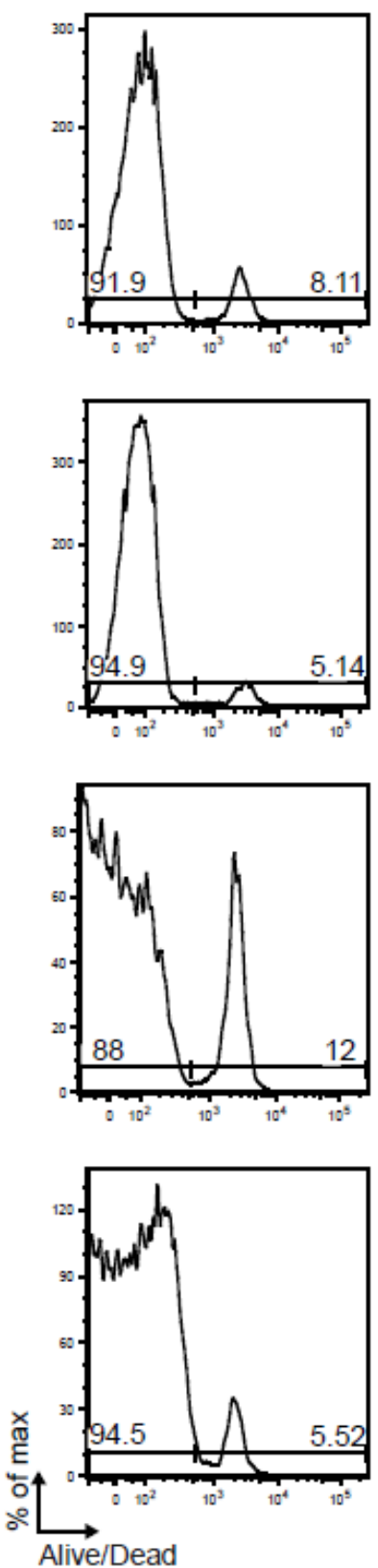

Fig. 6. BAFF partially reverses B cell death induced by BambL. (A) Purified splenic MFo B cells from WT mice were stimulated with the indicated concentrations of BambL. Cell viability was assessed after 24 hours in parallel with that of an unstimulated control by flow cytometric analysis using the viability dye. Data are means \pm SD from three independent experiments and were analyzed by Student's t-test to assess statistical significance. (B) Purified splenic MFo B cells from WT mice were stimulated ex vivo in the presence of $2.5 \mu \mathrm{g} / \mathrm{ml} \mathrm{BambL}$ with or without BAFF co-stimulation. The cells were analyzed 24 hours later by flow cytometry to generate FSC and SSC plots and viability dye histograms. Cells negative for the viability dye were considered to be alive, whereas those cells positive for the dye were considered to be dead. Data are representative of four independent experiments. (C) Flow cytometric analysis of the binding of BambL-Cy5 to MFo B cells in the presence and absence of soluble BAFF $(100 \mathrm{ng} / \mathrm{ml})$. Data are representative of three independent experiments. 
A

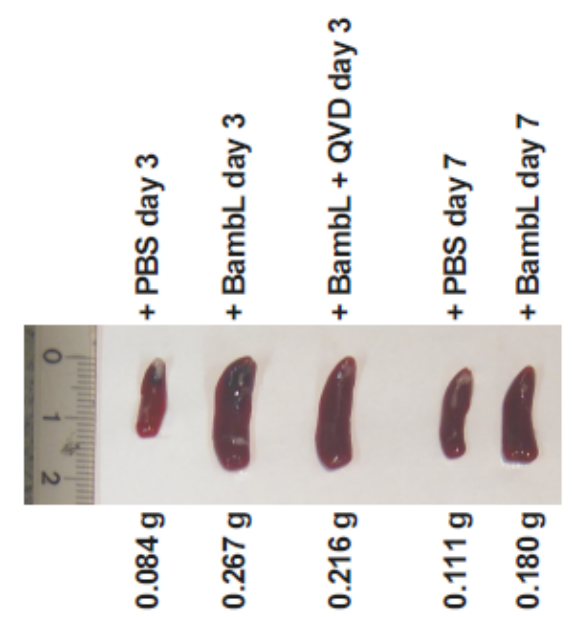

$C_{\mathrm{sP}}$

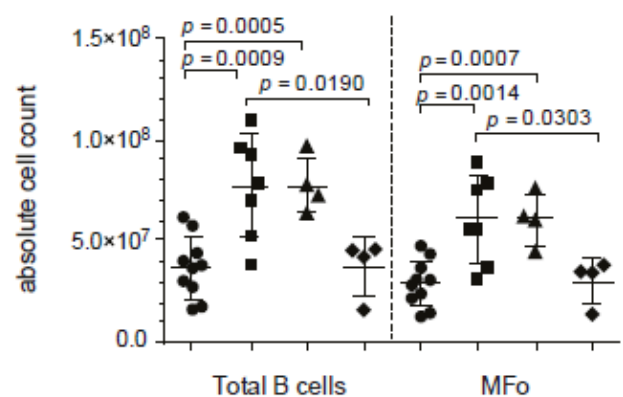

E

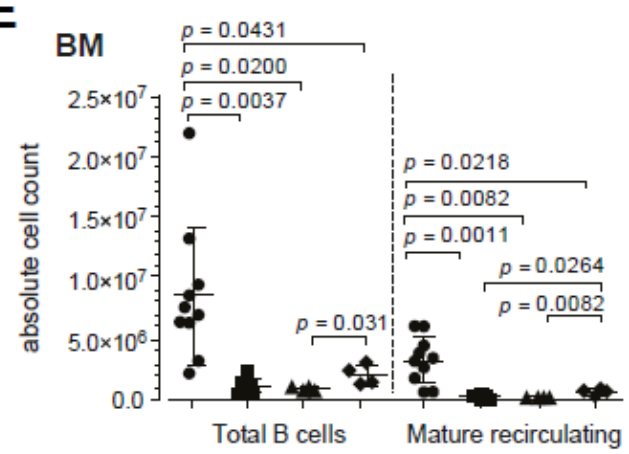

B

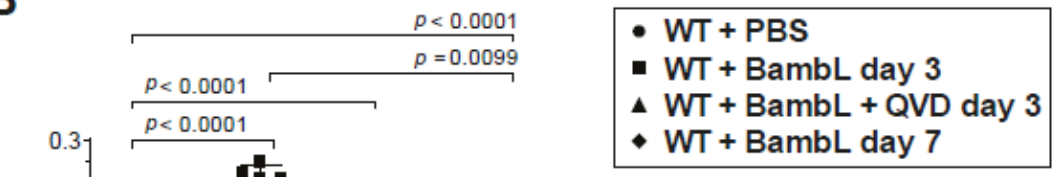

$D_{S P}$
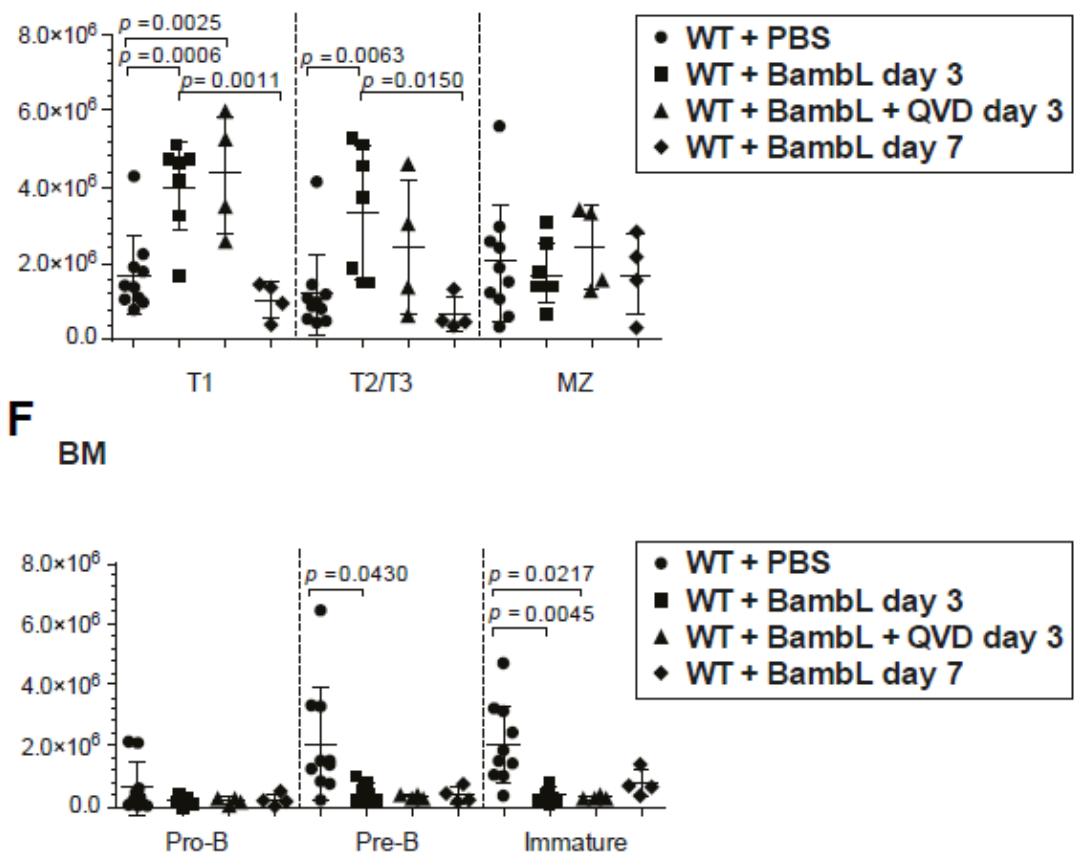

Fig. 7. Intraperitoneal injection of mice with BambL leads to splenomegaly and accumulation of B cells in the spleen with simultaneous lymphopenia in the bone marrow. (A) Images of spleens for the indicated times and treatment conditions after mice were injected with BambL. The sizes of the spleens were directly compared after extraction. Scale bar is given in $\mathrm{cm}$. (B) Statistical analysis of spleen weights from the indicated groups of treated mice. (C and D) Statistical analysis of the numbers of the indicated B cell populations in the indicated groups of treated mice. ( $\mathbf{E}$ and $\mathbf{F})$ Absolute counts of the indicated B cell populations in the bone marrow of the indicated groups of treated mice. In (B) to (F), each dot indicates an individual mouse: PBS, $n$ $=10 ;$ BambL d3, $n=7 ;$ BambL + QVD d3, $n=4 ;$ BambL d7, $n=4$. The data represent a compilation of values from four to seven independent experiments. All $P$ values were obtained using a two-tailed Student's t-test. 

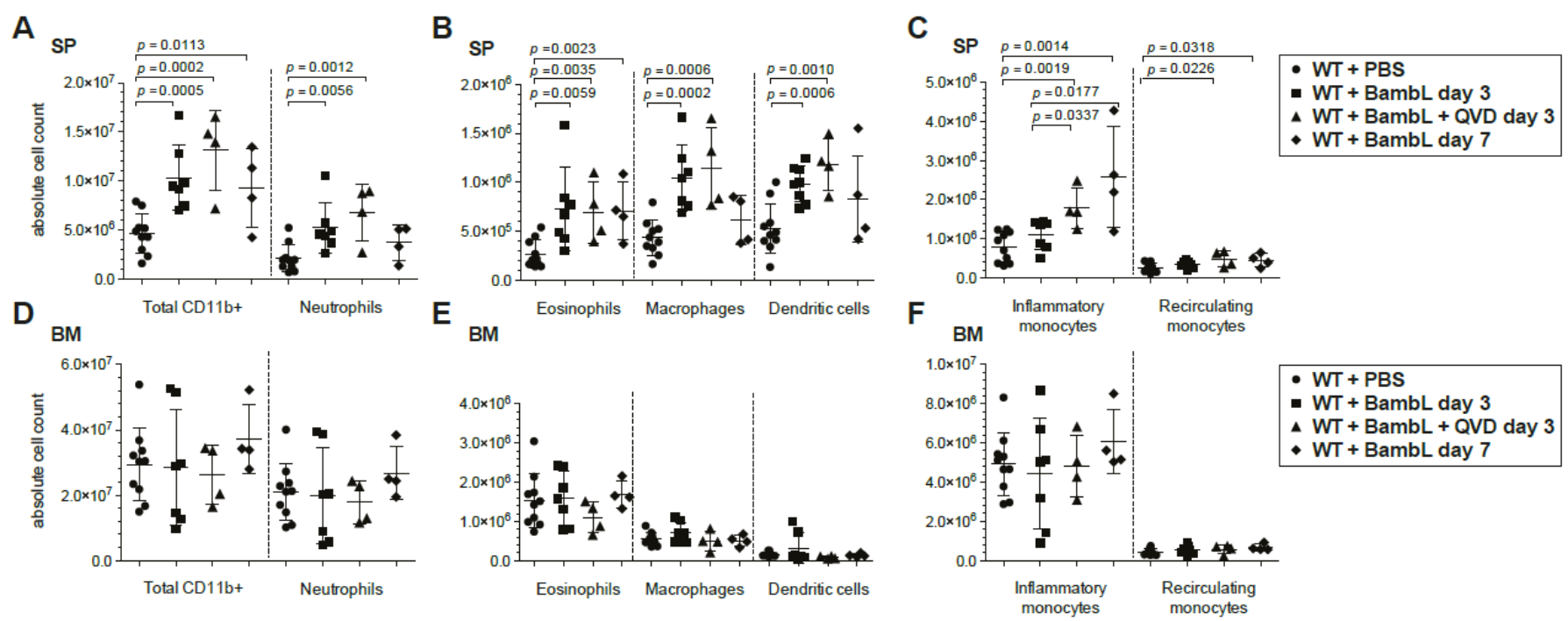

Fig. 8. Myeloid cell counts are increased in mice after injection with BambL. (A to C) Absolute counts of the indicated myeloid cell populations in the spleens of mice from the indicated treatment groups. (D to F) Absolute counts of the indicated myeloid cell populations in the bone marrow of mice from the indicated treatment groups. In (A) to (F), each dot indicates an individual mouse: PBS, $n=10$; BambL d3, $n=7$; BambL + QVD d3, $n=4 ;$ BambL d7, $n=4$. The data represent a compilation of values from four to seven independent experiments. All $P$ values were obtained using a two-tailed Student's t-test. Gating strategy was performed as described previously $(67,68)$. 